\title{
Image dipoles and polarons in organic semiconductors
}

Yongzhen Chen 



\title{
Image dipoles and polarons in organic semiconductors
}

\author{
Yongzhen Chen
}

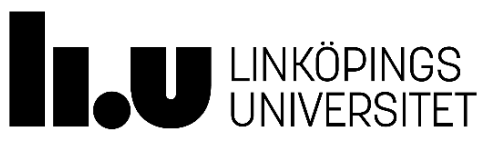

Department of Science and Technology

Faculty of Science and Engineering

Linköping University, SE-581 83 Linköping, Sweden

Norrköping 2021 
(c) $\mathrm{EY}$ This work is licensed under a Creative Commons Attribution 4.0 International License.

https://creativecommons.org/licenses/by/4.0

Image dipoles and polarons in organic semiconductors

Copyright (C) Yongzhen Chen, 2021

ISBN: 978-91-7929-061-o (print)

ISBN: 978-91-7929-062-7 (PDF)

ISSN: 0345-7524

Printed in Sweden by LiU-tryck, Sweden 2021 


\begin{abstract}
The rapid development of organic electronics depends on the synthesis of new $\pi$-conjugated molecules/polymers and the exploration of fundamental physics. However, most of the efforts have been concentrated on the former, leading to a lack of thorough understanding of many important concepts, which will become the ultimate limiting factor for overall performance and applications. Thereinto, the interface energetics in multilayer stacked optoelectronic devices and the electronic structure of doped organic semiconductors are two of the most complicated and yet inevitable topics in this field. A better understanding of them can provide needed additional insights into the operation of devices and give valuable guidance for device and molecule design. Hence, the aim of this thesis is to investigate these two fundamental issues using various spectroscopic characterizations and supported by computational modeling.

The cathode/organic interface plays a critical role in achieving balanced charge transport and improved stability in organic electronic devices. Employing stable cathode materials, however, typically results in large electron injection barriers and sub-optimal devices. Using small-molecule electron transport materials (ETMs) as interlayers is an effective approach to reduce the electron injection barriers, but the working mechanism is still under debate. By studying the energy level alignment behavior of ETMs on different types of substrates, we find the work function of the substrate is reduced by an extra "image" dipole formed at the interface and within the first layer of the ETM film. The use of non-reactive substrates and the results from X-ray photoelectron spectroscopy core level analysis exclude the orbital hybridization theory, which states that an ETM-metal complex may form at the interface. The characterization results on molecular orientation disqualify an explanation using intrinsic molecular dipole moments. Instead, experiments demonstrate that the interface dipole depends on the areal density and direction of lone electron pairs on the heteroatoms in ETMs, which is similar to previous observations in tertiary aliphatic amines. This behavior is well described by the so-called "double dipole step" model, where one dipole formed by the nitrogen nuclei and the lone pairs in the organic side points from the substrate surface to the organic film and the other one formed by their image charges in the electrode side shows the same direction.
\end{abstract}

The polaron charge carrier is another important concept involved in multiple (opto)electronic processes during device operation, such as charge transport, exciton recombination/dissociation. Although numerous experimental and theoretical efforts have been made, there is still a lack of comprehensive studies on the electronic structure of negative polarons due to their high air sensitivity, including correlation between the valence 
band structure measured by ultraviolet photoelectron spectroscopy (UPS) and the optical band gap derived from the UV-vis-NIR absorption. In the present work, we are able to integrate the optical and electrical measurements with photoelectron spectroscopy to collect all information without breaking an ultra-high vacuum. Negative polarons formed in alkali metal-doped polymers are detected with new polaronic states below the Fermi level and lower energy absorption bands arising from the excitation from polaronic states to unoccupied states. In addition, the Fermi level shifts toward the conduction band with increasing the doping ratio, and the doubly-occupied polaronic state shows slightly lower energy than the topmost valance band peak of the neutral polymer. These observations are supported by the density functional theory (DFT) simulations, from which we also demonstrate that polaron pairs rather than bipolarons are preferentially formed at high doping ratios. By comparison of different polymer and dopant systems, we find the polymer-dopant interaction and the polaron delocalization are dependent on the distortion of the polymer backbone and the size of the dopant, properties that in turn affect the conductivity and air stability of the n-doped materials.

I hope that the findings presented in the thesis can greatly promote the understanding of the energetics of the ETMs/cathode interface and the electronic structures of negative polarons in alkali metal-doped polymers, contributing to further providing new guidelines for the molecular design and improve the device performance. 


\section{Populärvetenskaplig Sammanfattning}

Den snabba utvecklingen av organisk elektronik har gett upphov till ett brett spektrum av applikationer, t.ex. organiska ljusemitterande dioder (OLED) för skärmar och belysning, organiska solceller för energiomvandling och organiska fälteffekttransistorer (OFET) för sensorer och logiska krets. De betydande framstegen är oskiljaktiga från syntesen av nya $\pi$ konjugerade molekyler/polymerer och utforskningen av den grundläggande fysiken bakom. Ansträngningarna har dock mest koncentrerats till det förstnämnda, vilket har lett till brister i den grundläggande förståelsen av många viktiga koncept, vilket begränsar arbetet med att förbättra organisk elektronikkomponenternas prestanda. Energinivåupplinjering vid gränsytor i optoelektroniska komponenter och mer generellt elektronstrukturen av dopade halvledare är två komplicerade områden för grundforskning som är av stor betydelse för komponent- och materialutveckling. En bättre förståelse här kan ge ytterligare insikter om hur material och komponenter fungerar och ge värdefull vägledning för komponent- och molekyldesign. Därför är syftet med denna avhandling att undersöka dessa grundläggande områden med hjälp av olika spektroskopiska karakteriseringar.

Katod/organiska -gränssnitt spelar en avgörande roll för att uppnå en balanserad laddningstransport och förbättrad stabilitet i elektroniska komponenter. Atmosfär-stabila katodmaterial medför ofta stora elektroninjektionsbarriärer vilket i sin tur försämrar komponenternas driftsstabilitet och prestanda. Även om elektrontransportmaterial med små molekyler (ETM) har använts i stor utsträckning som katodskikt för att minska injektionsbarriären i OLEDs och solceller, är deras arbetsmekanism fortfarande oklar. Genom att studera energinivåjusteringsbeteendet för ETM på olika typer av substrat, finner vi att substratets utträdesarbete reduceras med en extra "spegel"-dipol som bildas vid gränsytan mellan ETM -filmen och katodytan. Användningen av icke-reaktiva substrat och analys av så-kallade spektroskopiska kemiska skift utesluter orbitalhybridisering, dvs att ETM-metallkomplex bildas vid gränssnittet. Mätresultat om molekylär orientering motsätter sig förklaringar baserade på kollektiva intrinsiska molekylära dipolmomentet. Istället visar experiment att dipolen vid gränsytan beror på ytdensitet och riktning för de ensamma elektronparen hos kväveatomerna i ETM, vilket liknar tidigare observationerna för tertiära alifatiska aminer. Detta beteende beskrivs väl av den så kallade "dubbelt dipolstegs modellen", där en dipol som bildas av kvävekärnorna och de ensamma elektronparen i det organiska lagret pekar från substratytan till den organiska filmen och inducerar en "spegelbilds"-dipol i elektrodytan som visar samma riktning. Dessa resultat hjälper till att bättre förstå ETM/elektrodgränssnittets funktion och möjliggör nya riktlinjer för den molekylära konstruktionen av katodskiktet. 
Laddningsbärande polaroner ingår i flera fotoelektriska processer under komponentdrift, t.ex. laddningstransport, exciton-rekombination/dissociation. Därför är fundamental förståelsen av deras natur av avgörande betydelse för att förbättra komponentprestanda. Även om många experimentella och teoretiska ansträngningar har gjorts saknas det fortfarande omfattande studier om den elektroniska strukturen hos negativa polaroner på grund av deras höga luftkänslighet, speciellt korrelationen mellan valensbandstrukturen mätt med ultraviolett fotoelektronspektroskopi (UPS) och det optiska bandgapet framtaget via UV-vis-NIR absorptionen. I det föreliggande arbetet kan vi integrera de optiska och elektriska mätningarna med fotoelektronspektroskopi för att samla all information utan att bryta (ultrahög) vakuum. Negativa polaroner som bildas i alkalimetall-dopade polymerer detekteras med nya polaroniska tillstånd under Fermi-nivån och lägre energiabsorptionsband som härrör från excitation från polaroniska tillstånd till ickeockuperade tillstånd. Dessutom skiftar Fermi-nivån mot ledningsbandet med ökande dopningsförhållande och det dubbelockuperade polaroniska tillståndet visar en något lägre energi än den högsta valensbandstoppen för den neutrala polymeren. Dessa observationer stöds av densitetsfunktionsteori (DFT) simuleringar, från vilka vi också visar att polaronpar snarare än bipolaroner företrädesvis bildas vid höga dopningsförhållanden. Genom jämförelse av olika polymer- och dopningssystem finner vi att polymerdopningsinteraktionen och polarondelokaliseringen är beroende av distorsion av polymerskelettet och dopjonens storlek, vilka i sin tur påverkar ledningsförmågan och luftkänsligheten av dopade filmer.

Jag hoppas att de resultat som presenteras i min avhandling främjar starkt förståelsen för hur ETM: er/katodgränssnittet fungerar och n-dopade organiska halvledare, med syfte att möjliggöra nya riktlinjer för molekylär designen och förbättrad komponentprestanda. 


\section{Acknowledgements}

This thesis is finished in the group of surface physics and chemistry after four years study and research. I would avail myself of this opportunity to express my great appreciation to those who have given me support and concern in the past. First, I would like to express my deepest gratitude to my supervisor Prof. Mats Fahlman, for providing me a chance to pursue my $\mathrm{PhD}$ study in the field of organic electronics. All study and research are inseparable from your continuous guidance, encouragement, and support. I have benefited so much from your profound knowledge, practical wisdom, and immense personal charm. I really appreciate the experience of being your student and working with so many outstanding people.

I would like to thank my co-supervisor Dr. Xianjie Liu, who leads me on this wonderful journey. Thank you for your careful guidance and unreserved support over the past four years. I am very grateful for your instructions on my experiment and for sharing your profound knowledge to me. I would also like to thank Dr. Slawomir Braun for your great help and guidance in laboratory equipment and doping experiments. My thanks also go to my mentor Jianwu Sun.

I appreciate the help from my current and previous group members. In particular, thank you Chuanfei for sharing your knowledge. I learned a lot from the discussion with you. Thank you Jiquan for unreservedly sharing your experience with me and giving me a lot of practical advice. Thanks to Qian Zhang, Yong Yu, Qilun, Xiane, Qingqing and Anna for your help in the lab, as well as the discussions on research and life. I enjoy working and studying with you and thank you all for your friendship and accompany.

I would like to thank all collaborators Igor Zozoulenko, Sarbani Ghosh, Simone Fabiano, Chi-Yuan Yang and Hanyan Wu for delivering your knowledge and experiences to improve our work. I also want to thank Tero-Petri Ruoko for sharing your optical knowledge and giving helpful suggestions on the absorption measurement. Thanks to Shula Chen for NIR photoluminescence measurement. Thanks to Suhao Wang for the discussion on the fabrication of photodetector.

I would also like to thank my colleagues and Chinese friends in IFM and LOE, not explicitly mentioned here. Special acknowledgements to Prof. Magnus Berggren, the leader of LOE, for providing a dense and pleasant academic atmosphere, to the lab crew team for your efforts on maintaining such a nice working environment and help with various issues, to the administrative team for all the help with practical things. 
I would like to thank Prof. Ying Wang from the University of Chinese Academy of Sciences, who introduced me to this group and gave me many good suggestions over the past four years.

Finally, I would like to express my sincere gratitude to my family, especially my parents, for your unconditional love and support all the time, to $\mathrm{Na}$, for your love, accompany and encouragement in every stressful moment. 


\section{List of Publications Included in this Thesis}

1. Image-Force Effects on Energy Level Alignment at Electron Transport Material/Cathode Interfaces

Yongzhen Chen, Xianjie Liu, Slawomir Braun, Ying Wang, and Mats Fahlman Journal of Materials Chemistry C, 2020, 8, 173-179

2. Understanding Interface Dipoles at Electron Transport Material/Electrode Modifier for Organic Electronics

Yongzhen Chen, Xianjie Liu, Slawomir Braun, and Mats Fahlman ACS Applied Materials \& Interfaces, 2021, 13, 47218-47225

3. Experimental and Theoretical Investigation into the Polaron Structure of K-Doped Polyfluorene Films

Yongzhen Chen, Sarbani Ghosh, Xianjie Liu, Igor V. Zozoulenko, Mats Fahlman, and Slawomir Braun

The Journal of Physical Chemistry C, 2021, 125, 937-945

4. In-situ Spectroscopic and Electrical Investigations into Ladder-Type Conjugated Polymers Doped with Alkali Metals

Yongzhen Chen, Han-Yan Wu, Chi-Yuan Yang, Xianjie Liu, Slawomir Braun, Simone Fabiano, and Mats Fahlman

Submitted

Author's contributions:

Did most of the experimental work and data analysis, wrote the first draft and responsible for the revision process and manuscript submission. 


\section{List of Publications not Included in this Thesis}

1. Nanocontacts Give Efficient Hole Injection in Organic Electronics

Qingzhen Bian, Chiara Musumeci, Chuanfei Wang, Andreas Skallberg, Yongzhen Chen, Zhangjun Hu, E. Peter Münger, Kajsa Uvdal, Mats Fahlman, and Olle Inganäs Science Bulletin, 2021, 66, 875-879

2. Highly Efficient White Light-Emitting Diodes with a bi-Component Emitting Layer Based on a Blue and Yellow Thermally Activated Delayed Fluorescence Emitters

Yongzhen Chen, Shengyong Li, Taiping Hu, Xiaofang Wei, Zhiyi Li, Yanwei Liu, Jianjun Liu, Ruifang Wang, Yuanping Yi, Cuihua Zhao, Ying Wang, and Pengfei Wang

Journal of Materials Chemistry C, 2018, 6, 2951-2956

3. N-Doping-Induced Efficient Electron-Injection for High Efficiency Inverted Organic Light-Emitting Diodes Based on Thermally Activated Delayed Fluorescence Emitter

Yongzhen Chen, Xiaofang Wei, Zhiyi Li, Yanwei Liu, Jianjun Liu, Ruifang Wang, Pengfei Wang, Yukiko Yamada-Takamura, and Ying Wang

Journal of Materials Chemistry C, 2017, 5, 8400-8407

4. Triplet Decay-Induced Negative Temperature Dependence of the Transient Photoluminescence Decay of Thermally Activated Delayed Fluorescence Emitter

Xiaofang Wei, Yongzhen Chen, Ruihong Duan, Jianjun Liu, Ruifang Wang, Yanwei Liu, Zhiyi Li, Yuanping Yi, Yukiko Yamada-Takamura, Pengfei Wang, and Ying Wang

Journal of Materials Chemistry C, 2017, 5, 12077-12084

5. Highly Conductive, Air-Stable Silver Nanowire@Iongel Composite Films toward Flexible Transparent Electrodes

Weiwei Xiong, Hongliang Liu, Yongzhen Chen, Meiling Zheng, Yuanyuan Zhao, Xiangbin Kong, Ying Wang, Xiqi Zhang, Xiangyu Kong, Pengfei Wang, and Lei Jiang

Advanced Materials 2016, 28, 7167-7172 


\section{Abbreviations}

OS

OLED

OPV

OFET

ICT

CPX

PES

XPS

UPS

IPES

NEXAFS

UV-Vis-NIR

SEM

HOMO

LUMO

IP

EA

$E_{g}$

$\mathrm{E}_{\mathrm{F}}$

$\Phi$

VL

$\Delta$

$\mathrm{E}_{\mathrm{k}}$

$\mathrm{E}_{\mathrm{B}}$

$\mathrm{E}_{\text {cutoff }}$

EICT+

EICT-

Eexciton

ETM

EL

HTL

ETL

CGL

SAM

EQE
Organic semiconductor

Organic light-emitting diode

Organic photovoltaic

Organic field-effect transistors

Integer charge transfer

Charge-transfer complex

Photoelectron spectroscopy

X-ray photoelectron spectroscopy

Ultraviolet photoelectron spectroscopy

Inverse photoelectron spectroscopy

Near edge X-ray absorption fine structure

Ultraviolet-visible-near infrared

Scanning electron microscope

Highest occupied molecular orbital

Lowest unoccupied molecular orbital

Ionization potential

Electron affinity

Band gap

Fermi level

Work function

Vacuum level

Vacuum level potential step

Kinetic energy

Binding energy

Secondary-electron cutoff

Positive pinning energy

Negative pinning energy

Exciton binding energy

Electron transport material

Emitting layer

Hole transport layer

Electron transport layer

Charge generation layer

Self-assembly monolayer

External quantum efficiency 
DFT

TD-DFT
Bandwidth

Polarization energy

Geometric relaxation energy

Degree of charge transfer

Density functional theory

Time-dependent density functional theory 


\section{Contents}

Abstract...........................................................................

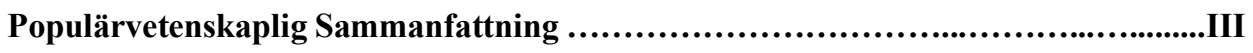

Acknowledgements.....................................................................V

List of Publications Included in this Thesis........................................VII

List of Publications not Included in this Thesis....................................VIII

Abbreviations........................................................................IX

Chapter 1 General introduction....................................................1

Chapter 2 Organic semiconductors...................................................5

2.1 Electronic structures of organic semiconductors................................6

2.2 Charge carriers in organic semiconductors.................................. 11

Chapter 3 Interfaces in organic electronics.........................................15

3.1 Common interfaces in optoelectronic devices................................. 15

3.2 Energy level alignment..................................................... 17

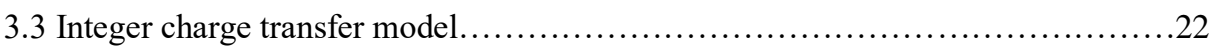

Chapter 4 Chemical doping in organic semiconductors............................25

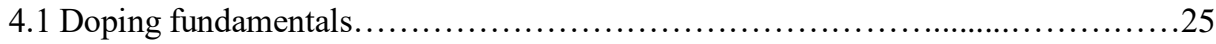

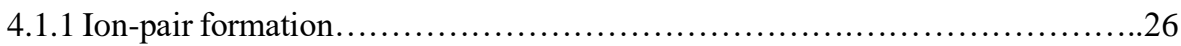

4.1.2 Charge-transfer complex formation.....................................27

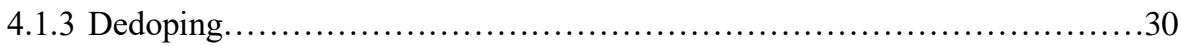

4.2 Dopants............................................................. 31

$4.2 .1 \mathrm{n}$-type molecular dopants.............................................. 32

4.2 .2 p-type molecular dopants............................................. 33

4.3 Applications of chemical doping in optoelectronic devices....................... 34

4.3.1 Doped layers in OLEDs.............................................. 35

4.3.2 Doped layers in OPVs.............................................. 37

Chapter 5 Photoelectron spectroscopy...........................................39

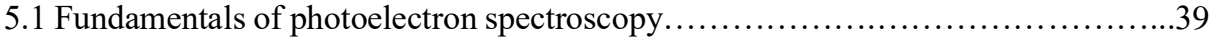

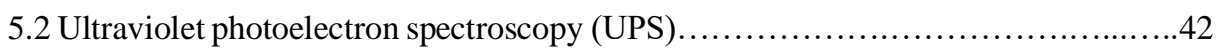

5.3 X-ray photoelectron spectroscopy (XPS) .................................... 43

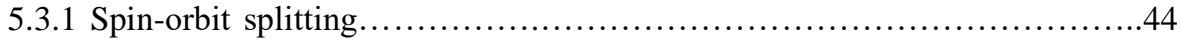

5.3.2 Chemical shifts........................................................... 44

5.3 .3 Shake-up and shake-off satellites.................................... 45

Chapter 6 Summary and outlook................................................47

6.1 Summary of papers.................................................... 47

6.1 .1 Paper I........................................................ 47 
6.1.2 Paper II............................................................. 49

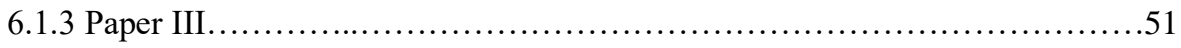

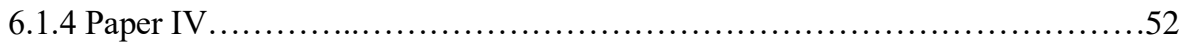

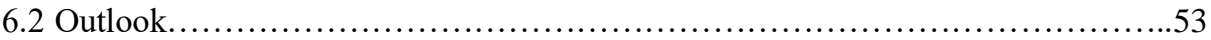

References...................................................................55

Publications............................................................................67 


\section{Chapter 1 General introduction}

Advances in the field of organic electronics are enabling their widespread applications in many areas, such as organic light-emitting diodes (OLEDs) for display and lighting, ${ }^{1-4}$ organic photovoltaics (OPVs) for energy conversion, ${ }^{5-7}$ and organic field-effect transistors (OFETs) for sensors and logical circuits. ${ }^{8-10}$ Thereinto, OLED-based products have been commercialized for more than 20 years and dominate today's industries such as upscale smartphones and televisions. OPVs and OFETs are also trying to find their way to becoming commercialized although their performance in some aspect requires further improvement. Compared with Si-based electronics, organic electronics thrives on the hope of enabling low-cost, solution-processed, flexible devices with advantageous mechanical, optical, and chemical properties. ${ }^{11-13}$ The realization of all these advantages benefits from the development of $\pi$-conjugated organic semiconductors (OSs), i.e., small molecules and polymers, whose chemical structure can be readily tuned toward application-specific demands. The function and application of the OSs depend on their photophysical properties both in ground and excited states, such as electrical conductivity, optical absorption, permittivity, (non)radiative transition rate, fluorescence quantum yield, etc., which are determined eventually by their chemical and electronic structures. ${ }^{14,15}$

All devices mentioned above are composed of single or multiple OS layers assembled with high conducting electrodes, e.g., metals or metal oxides. Accordingly, several interfaces will be formed during the growth of these layers, which play critical role in the operation of the device, especially for the electrode/OS interface that determines the charge injection/extraction process. ${ }^{16-19}$ A universal method to reduce the barrier and improve the charge injection/extraction is to insert an interlayer between the electrode/OS. On the cathode side, inorganic salts have been widely used both in OLEDs and OPVs, and have been proven to form a dipole layer at the electrode surface. ${ }^{20,21}$ Polymeric dipole layers, such as polyethyleneimine (PEI), poly[(9,9-bis(3'-(N,N-dimethylamino)propyl)-2,7fluorene)-alt-2,7-(9,9-dioctylfluorene)] (PFN) are developed to be used in the solutionprocessed and inverted devices. ${ }^{22,23}$ The small-molecule electron transport materials (ETMs), such as bathophenanthroline (BPhen), bathocuproine (BCP) are also usually used directly as the electron injection layer. ${ }^{24-26}$

The electronic structure of an organic solid is generally localized on individual molecules or polymer chains with weak intermolecular interactions by van der Waals forces, 
resulting in the different energetics at the electrode/OS interface with respect to the inorganic semiconductors and metal conductors. ${ }^{27,28}$ Thus, understanding of the energy level alignment at the electrode/OS interface has become one of the most complicated and key issues for the organic optoelectronic device. ${ }^{28,29}$ Even though many efforts have been made to model and predict such interfaces, none of them is suitable for all cases. The difficulties come mainly from the build-in complexity of organic systems, such as the various electronic structures caused by heteroatoms, chemical defects introduced during synthesis and different local order.

In the case of weakly interacting interface by physisorption, the integer charge transfer (ICT) model was proposed and validated by numerous metal/OS and OS/OS interfaces. ${ }^{30,31}$ It applies to most interfaces of electronic devices that formed by thermal sublimation and solution process of OSs on passivated substrates. The existence of the ultra-thin inert layer allows the interaction between the substrate and OS only by integer electron tunnelling under certain conditions. Interfacial polarons produced in this process feature new states, i.e., ICT states in the energy gap due to the electrostatic coupling of the substrate image charges. The resulting energy level alignment predicted by the ICT model is divided into three regimes: (i) Fermi level is pinned at the edge of negative ICT state, EICT-, with an upshift potential step when the work function of the substrate is smaller than $\mathrm{E}_{\text {ICT-}}$; (ii) Vacuum level alignment with negligible interface dipole when the work function of the substrate is between $\mathrm{E}_{\mathrm{ICT}-}$ and $\mathrm{E}_{\mathrm{ICT}+}$; (iii) Fermi level of the substrate is pinned at the edge of positive ICT state, $\mathrm{E}_{\mathrm{ICT}+}$, with a downshift potential step when the work function of the substrate is greater than $\mathrm{E}_{\mathrm{ICT}+}$. The ICT model predicts the smallest injection barrier at the electrode/OS interface and provides a guidance for the selection of the electrode material.

Polarons not only are present at the interface described above, but are also a key concept in the field of organic electronics and participates in many photoelectric processes, e.g., charge transport, exciton recombination/dissociation. ${ }^{32-34}$ Therefore, understanding the electronic structure and photophysical properties is of vital importance for improving device performance. Chemical doping is an effective approach to improve the electrical conductivity by introduction of negative (n-doping) or positive (p-doping) charges to the polymer chain. ${ }^{35-37}$ Meanwhile, it is also an important tool to investigate the charged states, i.e., polarons, of conjugated polymers. ${ }^{38-40}$ For polymers in neutral state, their transport gap is usually larger than $1.5 \mathrm{eV}$ and the density of intrinsic charge carriers is ultralow at room temperature, resulting in the poor electrical performance and limited application in the electronic devices. Doping induces electrons transfer to the lowest unoccupied molecular orbital (LUMO) or removal from the highest occupied molecular orbital (HOMO) of the polymer, producing new occupied or unoccupied spin-resolved energy levels in the band 
gap. ${ }^{19,41}$ The change in electronic structure increases free charges, i.e., electrons or holes, and yields new sub-gap transitions in the optical spectra.

The characterization work of this thesis is mostly based on photoelectron spectroscopy, including ultraviolet (UPS) and X-ray (XPS) photoelectron spectroscopy. This surface sensitive technique allows to probe the physical and chemical interactions at the surfaces and interfaces of the OS films in a relatively non-destructive manner. ${ }^{42}$ Thereinto, UPS focuses on the valence electronic structure by using a low energy photon source with a relatively high surface sensitivity and high resolution, directly providing the work function, the occupied density of states, the vertical ionization potential (IP) and the pinning energies. XPS focuses on core levels by using a high energy photon source, quantitatively detecting the proportion of atoms and chemical states. From the comparison of the spectra with and without OS layer, the energy level alignment is obtained upon the formation of the interface. The gap states formed by the polaron can also be detected near the Fermi level.

The first two works involved in this thesis explore the energy level alignment of the electrode/ETM interfaces. We observe the interface dipoles when ETMs are deposited on a series of conducting substrates and demonstrate the thickness-dependence of such dipoles. By studying the influence of intermolecular hydrogen bond and molecular orientation, the origin of the dipole is discussed in detail. We investigate the electronic structures of the charged polymers through the last two works. The evolution of work function, valence band, core levels, optical absorption and the electrical conductivity upon alkali metal doping is explored systematically. This thesis promotes the understanding of the energetics of the ETMs/cathode interface and the electronic structures of negative polarons in alkali metaldoped polymers.

The present chapter is a brief overview of the basic concepts involved in this thesis. What follows is the physical and chemical properties of OSs in chapter 2, a general description of interfaces in organic electronics in chapter 3 , fundamentals of chemical doping in chapter 4 , an introduction of the principle of the photoelectron spectroscopy technique in chapter 5 . In the last chapter, a summary of all papers included in this thesis is given. 


\section{Chapter 2 Organic semiconductors}

Organic semiconductors (OSs) are a class of semiconductor materials composed of carbon and hydrogen atoms, some of which contain a few heteroatoms such as sulfur, oxygen, and nitrogen. Different from the traditional inorganic semiconductors such as silicon, germanium and GaAs, whose intrinsic conductivity can reach $10^{-8}-10^{-2} \mathrm{~S} \mathrm{~cm}^{-1}$ by thermal excitation at room temperature, the pure OSs are almost non-conductive with negligible free charge due to the lower dielectric constants and larger transport gap (1.5-3.0 eV). Their conductivity is extrinsic and can be caused by the electrical or optical excitation, by impurities left from synthesis or induced by the atmosphere, or by intentional doping. Early research about OSs is concentrated on the electroluminescence of small molecular crystals. ${ }^{43,44}$ The progress has been speeded up by the discovery of high conductive $\pi$ conjugated polymers in the late 1970s and taken off with the report of the OLED on thin amorphous films by C. W. Tang in 1987.45,46 The device structure used in that report becomes a prototype of all current devices.

OSs usually consist of three varieties, i.e., amorphous molecular film, molecular crystal, and polymer film. The amorphous molecular films are mostly deposited by thermal evaporation method, some are also by spin-coating method. They are widely used in OLEDs and the small-molecule OPVs. Different from the atomic and ionic crystals, molecules such as naphthalene and anthracene form organic crystals through weak van-der-Waals interactions. The high mobility of the crystals enables their application in transistors. ${ }^{47,48}$ Polymer films are usually processed from solutions, which allows the use of a series of deposition techniques, such as ink-jet printing, spin-coating, spray-coating, or roll-to-roll coating. This affords the realization of large area, flexible and low-cost OLEDs and OPVs. The type of OS is related to the electronic structures and further affects the photophysical properties.

All OS materials used in this thesis are list in Figure 2.1. Thereinto, bathophenanthroline (BPhen) and Bis-4,6-(3,5-dipyridylphenyl)-2-methylpyrimidine derivatives (i.e., B2PyMPM, B3РyMPM, B4PyMPM) are small molecules with relatively large band gap. They are usually processed by thermal evaporation and spin-coating methods and used as amorphous films. Due to their good electron mobility, they are usually used as the electron transport layer in OLED and OPV and also used as the interlayer of cathode to improve the electron injection/extraction. ${ }^{49-52}$ The polymer poly(9,9dioctylfluorene) (PFO), polybenzimidazobenzophenanthroline (BBL) and 
poly(bis(benzimidazo)benzophenanthroline-dione) (BBB) are used to study the electronic structures of polaron. They are prepared by spin-coating or spray-coating methods. PFO shows two well-defined conformations, i.e., distorted and planar backbones, which enables to detect the influence of conjugation length on the polaron. ${ }^{53} \mathrm{BBL}$ is a rigid planar polymer that can maximum the delocalization of the polaron. The semi-ladder polymer BBB is used as the reference of BBL.

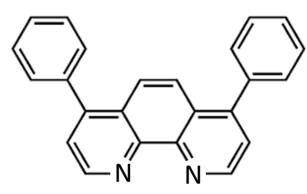

BPhen<smiles>Cc1nc(-c2cc(-c3ccccn3)cc(-c3ccccn3)c2)cc(-c2cc(-c3ccccn3)cc(-c3ccccn3)c2)n1</smiles>

B2PyMPM<smiles>Cc1nc(-c2cc(-c3cccnc3)cc(-c3cccnc3)c2)cc(-c2cc(-c3cccnc3)cc(-c3cccnc3)c2)n1</smiles>

B3РyMPM

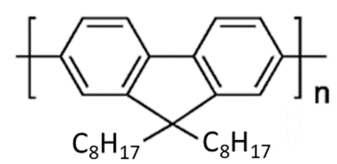

PFO

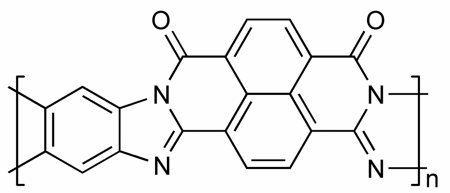

BBL<smiles>Cc1nc(-c2cc(-c3ccncc3)cc(-c3ccncc3)c2)cc(-c2cc(-c3ccncc3)cc(-c3ccncc3)c2)n1</smiles>

B4PyMPM

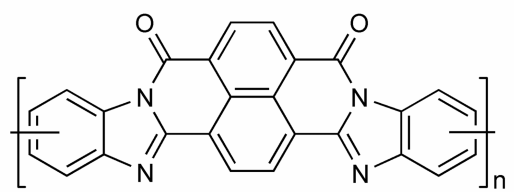

BBB

Figure 2.1 Chemical structures of OSs used in this thesis.

\subsection{Electronic structure of organic semiconductors}

Since the OSs are carbon-based materials, we start with the atomic orbitals of carbon. The electron configuration of carbon at ground state is written as $1 \mathrm{~s}^{2} 2 \mathrm{~s}^{2} 2 \mathrm{p}_{\mathrm{x}}{ }^{1} 2 \mathrm{p}_{\mathrm{y}}{ }^{1}$ with two singly occupied $2 \mathrm{p}$ orbitals. When it is bonding with other atoms such as hydrogen or another carbon, one electron will be excited from the low-energy $2 \mathrm{~s}$ orbital to the high-energy $2 \mathrm{p}_{\mathrm{z}}$ orbital and form four equivalent singly occupied orbitals that can contribute to the chemical 
bonding. The energy required for elevating the electron can be compensated by the energy released by the bond formation. In addition, the $2 \mathrm{~s}$ orbital will combine linearly with the $2 \mathrm{p}$ orbitals to form new hybrid orbitals, i.e., $\mathrm{sp}, \mathrm{sp}^{2}$ and $\mathrm{sp}^{3}$, depending on the number of covalence bonds that will be formed, as shown in Figure 2.2. When one $2 \mathrm{~s}$ orbital is mixed with three $2 \mathrm{p}$ orbitals, it will form four equivalent $\mathrm{sp}^{3}$ hybrid orbitals with an orbital angle of $109.5^{\circ}$. If the $2 \mathrm{~s}$ orbital is mixed with two $2 \mathrm{p}$ orbitals $\left(2 \mathrm{p}_{\mathrm{x}}\right.$ and $\left.2 \mathrm{p}_{\mathrm{y}}\right)$, it will form three $\mathrm{sp}^{2}$ orbitals. They are in the same plane with an angle of $120^{\circ}$ between them, leaving a $2 \mathrm{p}_{\mathrm{z}}$ orbital perpendicular to this plane. Two sp orbitals are formed in a line if the 2 s orbital is mixed with only one $2 p$ orbital. The remaining $2 p$ orbitals are respectively orthogonal to the hybrid orbitals.

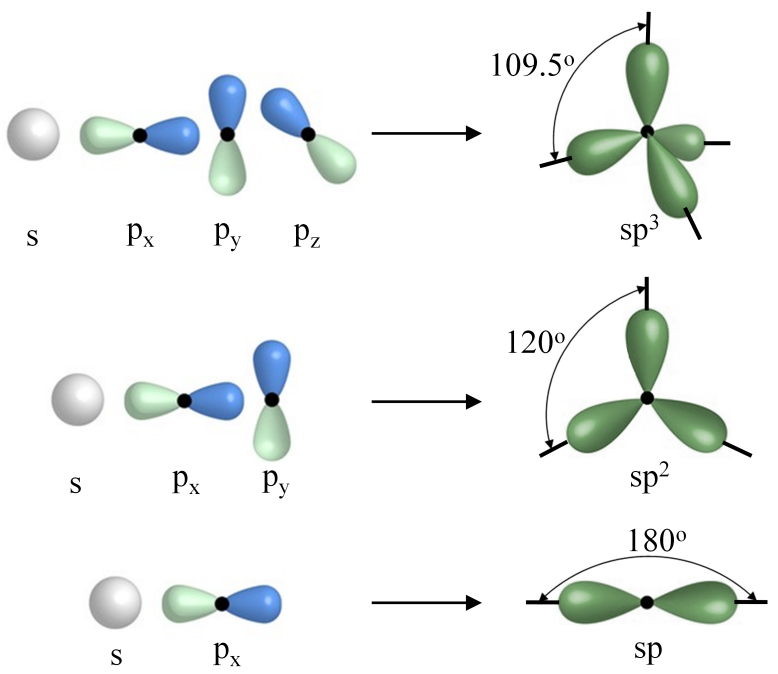

Figure 2.2 The atomic $\mathrm{s}$ and $\mathrm{p}$ orbitals and the formation of $\mathrm{sp}, \mathrm{sp}^{2}, \mathrm{sp}^{3}$ hybrid orbitals.

After bonding with other atoms, the molecular orbitals need to be considered instead of the atomic orbitals. In a double bond system, three $\mathrm{sp}^{2}$ orbitals overlap head-to-head with the orbitals in the neighboring atoms such as carbon and hydrogen to form three $\sigma$-bonds within a plane, that is, the planar skeleton. The unhybridized $2 \mathrm{p}_{\mathrm{z}}$ orbital orthogonal to the plane overlaps with another $2 \mathrm{p}_{z}$ orbital side-by-side to form the $\pi$-bond, where the electron density is concentrated above and below the planar skeleton. Meanwhile, anti-bonding orbitals, e.g., $\sigma^{*}$ and $\pi^{*}$, with higher energies are also formed during the linear combination between the atomic orbitals, as illustrated in Figure 2.3a. One electron from the $\mathrm{sp}^{2}$ orbital of the carbon and the other one from the same orbital of the neighboring carbon form an electron pair. The low-energy $\sigma$-orbital will be occupied by this electron pair to form the bonding orbital, leaving the high-energy $\sigma^{*}$-orbital empty to form the anti-bonding orbital. 
The electron pair from two $2 \mathrm{p}_{z}$ orbitals of the adjacent carbons fills the bonding $\pi$-orbital and leaves the anti-bonding $\pi^{*}$-orbital empty.

(a)

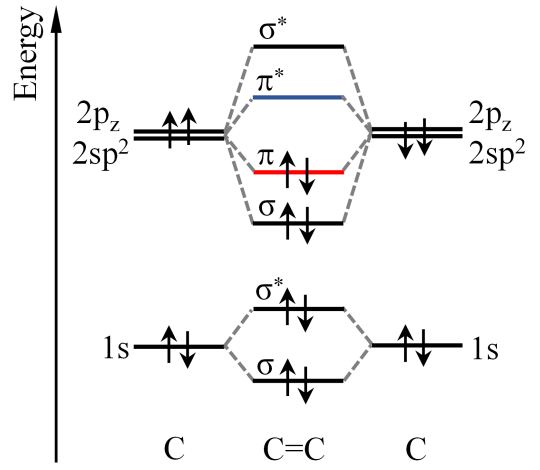

(b)

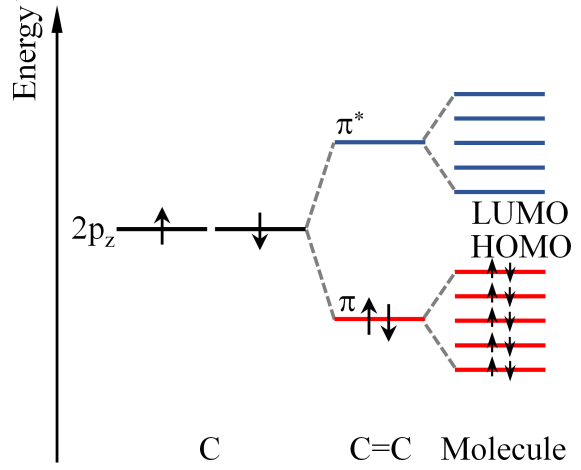

Figure 2.3 (a) An illustrate of the formation of $\sigma$ and $\pi$ bonds between two carbon atoms. (b) The formation of the occupied and unoccupied orbitals in an OS molecule.

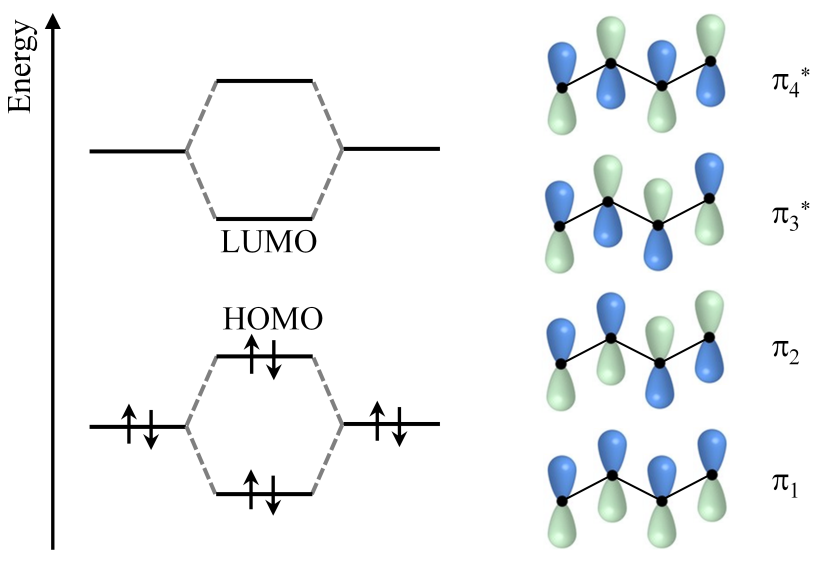

Figure 2.4 $\pi$-orbitals in conjugated butadiene. There are four orbitals with two bonding orbitals and two anti-bonding orbitals.

For $\pi$-conjugated molecules and polymers with alternating single and double bonds, $\pi$ orbitals are no longer isolated. They will interact with other neighboring $\pi$ orbitals to form a conjugated system. For example, $\pi-\pi$ conjugation in a butadiene molecule with four $2 p_{z}$ orbitals will form two occupied $\pi$-orbitals and two unoccupied $\pi *$-orbitals, as illustrated in Figure 2.4. With the increase of the conjugation length, the number of $\pi$ and $\pi^{*}$ orbitals 
increases, and the energy difference between the frontier orbitals HOMO and LUMO, i.e., transport gap, becomes smaller, as shown in Figure 2.3b. It enables the molecule or polymer to feature semiconductor characteristics. With the concepts of HOMO and LUMO, the ionization potential (IP) and electron affinity (EA) are easy to understand. IP is defined as the minimum energy required to remove an electron from the molecule/polymer to form a fully relaxed state, that is, from the HOMO to the vacuum level. While EA is defined as the maximum energy released to add an electron to the molecule/polymer to form a completely relaxed state, that is, from the vacuum level to the LUMO. ${ }^{54}$

So far, we have discussed the electronic structures of individual molecules. When molecules or polymers are in solid films, there will be additional intermolecular interactions, such as the $\pi-\pi$ stacking, which can significantly affect their photophysical properties. In a molecular crystal, the interaction between the frontier orbitals can create an energy band, i.e., valence band and conduction band, which is the prerequisite for realizing band transport, as shown in Figure 2.5. ${ }^{55}$ Since the intermolecular interactions are much weaker than the electronic coupling in inorganic crystals or polycrystals, the energy bands formed in molecular crystals are rather narrow (i.e., $w<0.3 \mathrm{eV}$ ). ${ }^{35}$ In the amorphous organic solid, which is also the most common situation, the electronic structures of OS molecules or polymer chains are typically defined by localized states. Their distribution and energy depend strongly on the local environment, e.g., the molecular arrangement and the nature of surrounding molecules/polymers. ${ }^{56,57}$ It results in the different polarization energies and broadens the distribution of the energy levels, which are usually assumed to follow a Gaussian distribution. ${ }^{58}$

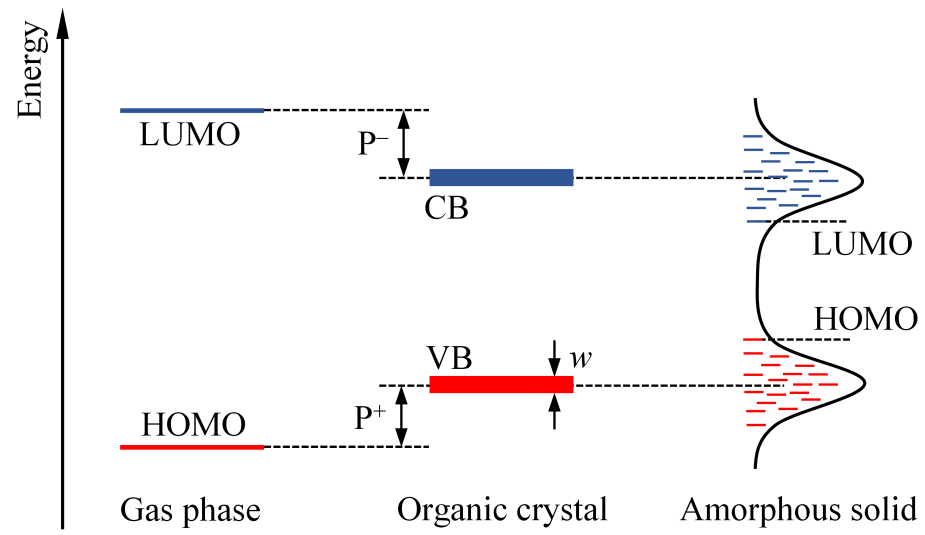

Figure 2.5 Energy level diagram of OS molecules in gas, crystal and amorphous solid. $\mathrm{P}^{+}$ and $\mathrm{P}^{-}$are polarization energies of cation and anion. $w$ is the bandwidth of the valence band (VB) or conduction band (CB). 
The IP, EA, and band gap $\left(\mathrm{E}_{\mathrm{g}}\right)$ of a molecule or polymer depend on their intended application and measurement technique. As shown in Figure 2.6, the first diagram shows the adiabatic energy levels, corresponding to the isolated non-ionized molecule. The lack of polarization effect results in a larger band gap compared to other scenarios. The second one shows the energy levels measured by UPS and inverse photoelectron spectroscopy (IPES). During the photoemission process, electrons are fully relaxed in the final state, resulting in the prominent shift of energy levels into the band gap, while the geometric relaxation ( $\lambda$, including molecule and lattice) does not have time to occur. Note that the electronic relaxation has a much greater contribution of more than $1 \mathrm{eV}$ compared to geometric relaxation. For instance, the IP of the top layer in the anthracene single crystal characterized by UPS is reduced by $1.2 \mathrm{eV}$ with respect to molecules in the vapor phase, and the value increases to $1.5 \mathrm{eV}$ for the second and subsequent layers. ${ }^{56}$ The third one refers to the fully relaxed charged state (i.e., polaron), where both the electronic relaxation and geometric relaxation are taken into account, resulting in a reduced transport gap. The total shift of the energy levels is defined as the polarization energy $\left(\mathrm{P}^{+}\right.$and $\left.\mathrm{P}^{-}\right)$and the difference between the photoemission and transport energy levels is the geometric relaxation energy $\left(\lambda^{+}\right.$and $\left.\lambda^{-}\right)$. The optical energy levels relate to the formation of excitons by photoexcitation or electroexcitation. The energy difference between the electrical transport gap and the optical band gap corresponds to the exciton binding energy ( $\left.\mathrm{E}_{\text {exciton}}\right)$, which is also an important concept both in OLEDs and OPVs.

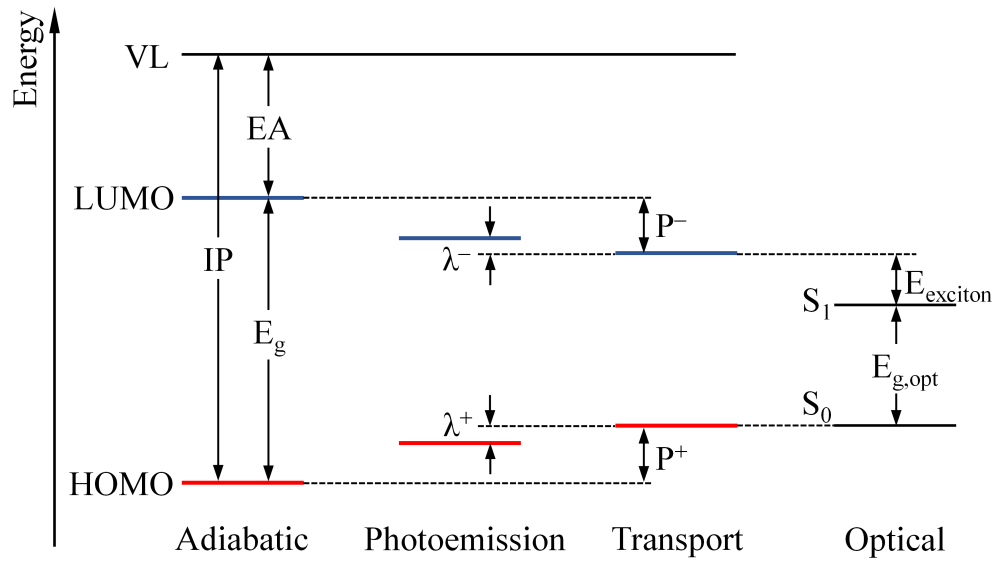

Figure 2.6 Schematic diagram of the relationship between different energy levels. The adiabatic scenario corresponds to the isolated non-ionized molecule. The photoemission scenario refers to the energy levels measured by UPS and IPES. The transport scenario is related to the fully relaxed charged state (i.e., polaron). The polarization energy (P) is composed of both electronic relaxation and geometric relaxation $(\lambda)$. The optical scenario is related to the excited state. 


\subsection{Charge carriers in organic semiconductors}

The electronic structure described above is only applied to OSs that are in the ground state. When OSs are under thermal, electrical, optical excitation, or chemical doping, free charges will be created with extra holes in the HOMO or electrons in the LUMO, resulting in the changes in both electronic and geometric structures of the OS. During this process, a quasiparticle, i.e., soliton or polaron is created. ${ }^{32}$ The former one is associated with the unpaired electron in the degenerate systems such as trans-polyacetylene and produced by the disruption of the local bond alternation. ${ }^{59}$ A soliton is neutral with a singly occupied orbital in the middle of the intrinsic polymer band gap. Addition or removal of an electron leads to the formation of spin-less negatively or positively charged soliton, as shown in Figure 2.7.

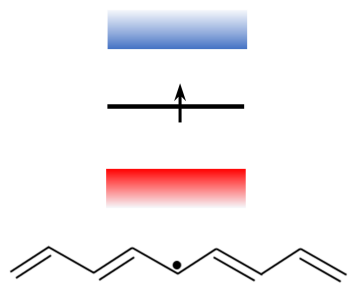

Neutral soliton

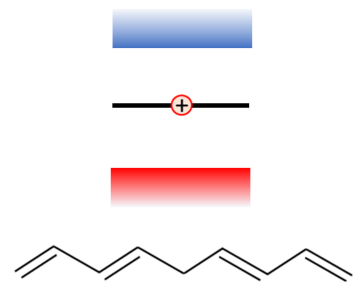

Positive soliton

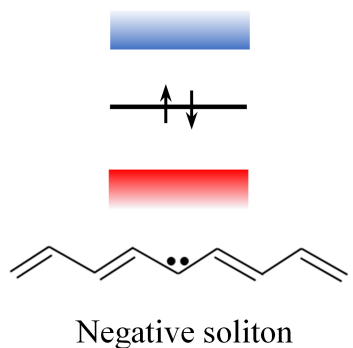

Negative soliton

Figure 2.7 The band structure of trans-polyacetylene containing (left) a positively charged soliton, (middle) a neutral soliton and (right) a negatively charged soliton.

The later one, polaron, is formed in the systems with a non-generate ground state, which applies to most conjugated polymers. A traditional model regarding to the polaron and bipolaron formation is shown in Figure 2.8. Adding an electron to the LUMO or removing an electron from the HOMO of the polymer chain will induce a large change in both electronic and geometric structures to obtain a fully relaxed state, i.e., polaron. It is delocalized over several repeat units. The new singly occupied state in the polaron is different from the energy of the HOMO or LUMO of the neutral polymer. Instead, it resides in the HOMO-LUMO gap and contributes to the sub-gap absorption transitions. ${ }^{60} \mathrm{~A}$ bipolaron is formed when the new state is doubly charged through the interaction of two polarons and is usually found, e.g., in the case of high doping level. ${ }^{60,61}$ 


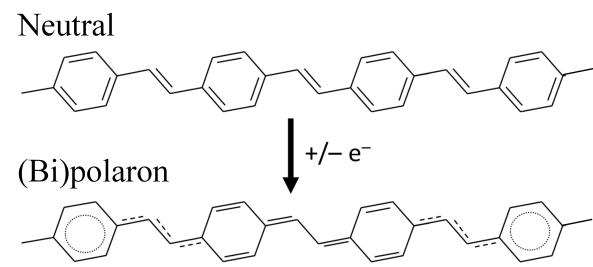

Charge and geometrical distortion

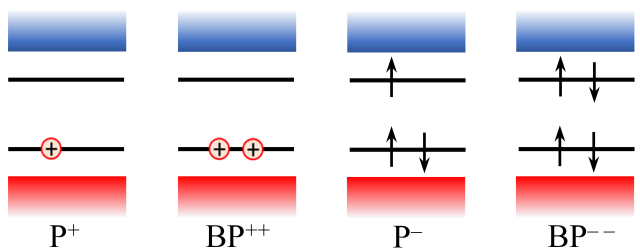

Figure 2.8 Schematic of chemical and electronic structure changes in the traditional model upon polaron and bipolaron formation. Adapted from Ref. 30.

However, this model is modified by the density functional theory (DFT) calculations in some recent reports, which brings a new interpretation of the polaron properties. ${ }^{62-64}$ Take the negative polaron as an example, the new singly occupied polaronic state in the model is replaced by an occupied spin-resolved state in the gap, accompanied by another unoccupied spin-resolved state that resides in the edge of the unoccupied levels, as shown in Figure 2.9. Meanwhile, the doubly-occupied state in the gap, which is formed by the shift of the original HOMO, is replaced by two occupied spin-resolved states in the edge of the occupied levels. The new energy level diagram results in several changes in the photoemission and optical transition. First, the singly occupied state described in the traditional model should be detected by both UPS and IPES, while the occupied spin-resolved state in the gap can only be observed by UPS. Second, the UPS feature from the doubly-occupied state in the traditional model should not change much compared to the neutral molecule/polymer, because this state is formed by the shift of the original HOMO into the band gap. However, it should show a significant change for the two occupied spin-resolved states with different energies. Third, due to the lack of empty states in the gap, no optical transitions are possible from the occupied levels to the polaronic level in the new diagram, which has been widely discussed in the traditional model. The case of positive polaron is also the same, which is shown in Figure 2.9c. 


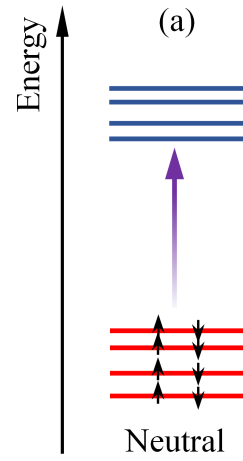

(b)

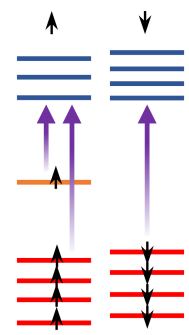

Negative polaron (c)

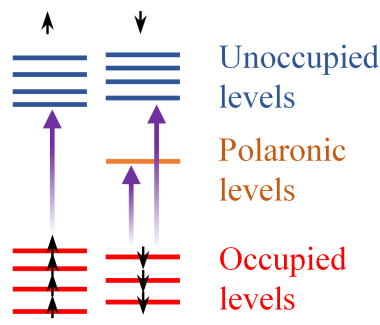

Positive polaron

Figure 2.9 Energy level diagrams of (a) neutral molecule/polymer, (b) negative polaron and (c) positive polaron based on the DFT calculations. The possible optical transitions are also involved.

Moreover, the modifications are also presented at high reduction or oxidation levels. The traditional model suggests that bipolarons ( $\operatorname{spin}=0$ ) will be formed when two electrons are added to the same molecule or the same site of a polymer chain. However, according to the DFT calculations, whether a bipolaron or a polaron pair (spin $=1$ ) is formed depends on the total energy of the system. If the exchange energy gained due to the increased total spin exceeds the electron pairing energy due to the electrostatic repulsion, a polaron pair is preferentially formed. Otherwise, a bipolaron is preferentially formed. For instance, calculations show that positively charged poly(3,4-ethylenedioxythiophene) (PEDOT) favors to form polaron pairs when the charge number is two $(\mathrm{Q}=2) \cdot{ }^{64}$ However, when the number of charges increases to 4 or 6 , it favors to form bipolarons for the PEDOT chain with 12 repeat units $(N=12)$ and polaron pairs for $N=18$. The spin is always $1 / 2$ when the number of charges is odd (i.e., 1, 3, 5). While the negatively charged PFO almost invariably form polaron pairs at high reduction levels, resulting in the high total spin, as shown in Figure 2.10. Even in the case of $Q=-6$, i.e., one electron per repeat unit, the spin is still not 0 , which means that PFO can be charged by more electrons.

The distribution of some orbitals in the neutral and negatively charged PFO chain is also shown in the figure, where the HOMO and LUMO of the neutral PFO are distributed on about 4.5 repeat units. However, they are completely changed upon the addition of one electron on the chain. The wave functions of these orbitals shrink to about 3 repeat units and the polaronic singly occupied state $\left(S_{\alpha}\right)$ appearing in the gap also shows a more localized characteristic compared to the descriptions in the traditional model, which makes it necessary to reconsider the Coulomb interaction created by the unpaired electron and results in the splitting of energy levels. 


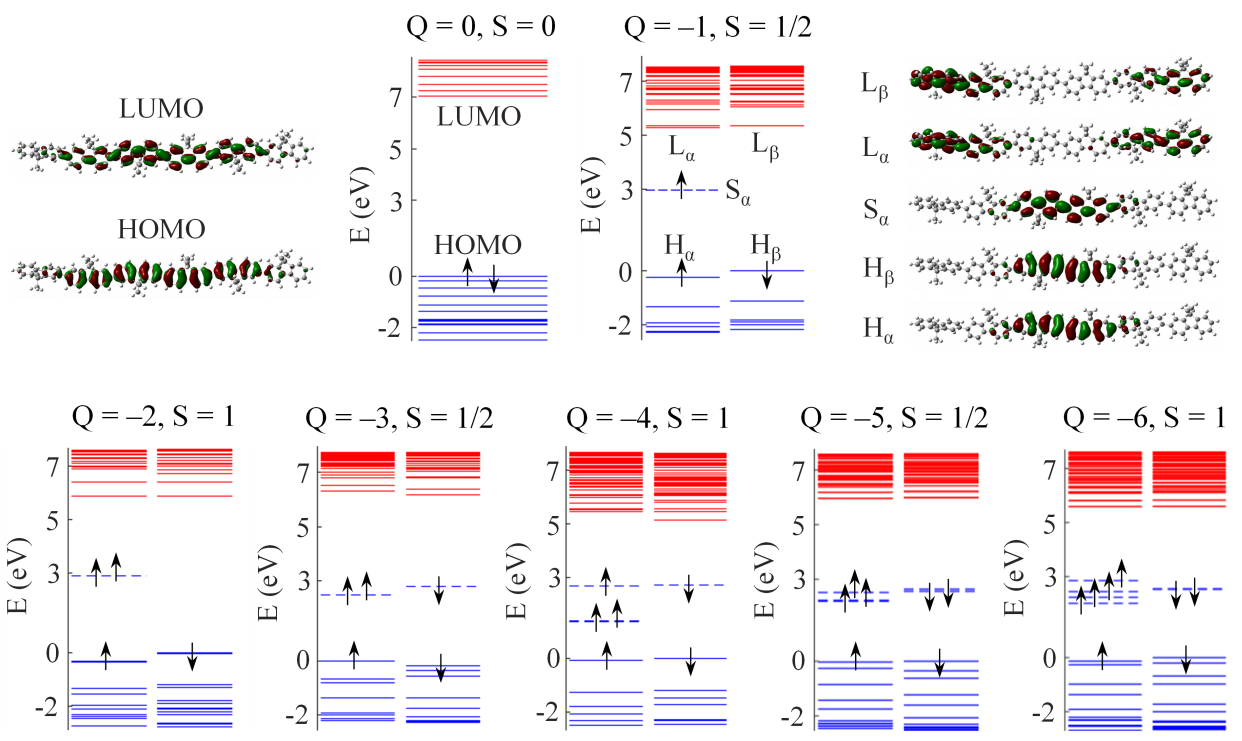

Figure 2.10 Energy level diagrams of neutral $(Q=0)$ and negatively charged $(Q=-1$ to 6) PFO chain with 6 repeat units. The distribution of $\mathrm{HOMO}\left(\mathrm{H}_{\alpha}, \mathrm{H}_{\beta}\right), \mathrm{LUMO}\left(\mathrm{L}_{\alpha}, \mathrm{L}_{\beta}\right)$ and SOMO $\left(\mathrm{S}_{\alpha}\right)$ in the neutral and one-electron charged chain $(\mathrm{Q}=0,-1)$ and the total spin at each reduction level are also involved.

The new diagram of the polaron and bipolaron has been verified by many experimental characterizations. Thereinto, the spin of negatively charged BBL predicted by the DFT calculation is consistent with the experimental observations, where it gradually rises as the reduction level increases from 0 to $100 \%$ and starts to drop when the reduction level is higher than $100 \%$ until it becomes 0 when the reduction level reaches $200 \%$. The maximum spin is $3 / 2$ at the reduction level of $100 \% .{ }^{63}$ The optical absorptions of the PEDOT and PFO evolving with the charge number predicted by the time-dependent DFT (TD-DFT) are also in agreement with the experimental results. ${ }^{64,65}$ In addition, the changes of the valence structure in the new diagram are more suitable for explaining the UPS spectra of alkali metal-doped PFO and BBL. These results suggest that the new diagram is more precise to describe the formation and properties of polarons. When approaching issues related to polarons, such as energy level alignment in the ICT model and charge injection/extraction barriers in the devices, the new energy level diagram will provide deeper understanding. 


\section{Chapter 3 Interfaces in organic electronics}

One of the critical physics behind the device operation is the interface properties both in inorganic and organic electronics. The electronic structure of interfaces between inorganic semiconductors has been well-interpreted by the band theory already in the last century. However, as an emerging technology in recent decades, the fundamental theory of interface properties in organic electronics is still being developed and refined after numerous experimental characterizations and theoretical simulations. ${ }^{30}$ The major obstacle can call back to the unique characteristics of OSs consisting of $\pi$-conjugated structures packed with weak intermolecular interactions. Besides, the complicated microstructure and versatile molecules greatly increase the study difficulty. This chapter aims to ravel out different types of common interfaces and their energetics, and discuss the roll of interfaces in optoelectronic devices.

\subsection{Common Interfaces in optoelectronic devices}

During the preparation of the organic optoelectronic devices, several interfaces will be formed upon the deposition of successive layers, e.g., between OSs and electrodes (metals or semimetals), between different OSs, between OSs and dielectrics, between dielectrics and electrodes, especially in the multilayer stacked OLEDs. As shown in Figure 3.1a, the basic functional layers of a traditional device structure successively are anode, hole transport layer (HTL), emitting layer (EL), electron transport layer (ETL) and cathode. With bias applied, holes are injected from the anode to HTL, while electrons are injected from the cathode to ETL. They are driven across the transport layer and recombine at the EL to form excitons. During this process, holes and electrons go through several interfaces with different potential barriers. To balance the charge transport and lower the operating voltage of the device, choosing materials with appropriate energy levels is usually more important than tuning the conductivity and thickness of the transport layers.

Another critical and effective method to lower the injection barrier is by employing an interlayer between the electrode and transport layer. Many efforts have been made to develop new interlayer materials and explore the interface energetics. In a traditional bottom-emitting OLED, the use of transparent metal oxides and poly(3,4ethylenedioxythiophene):poly(styrenesulfonate) (PEDOT:PSS) as anodes provides a high work function to match the HOMO of many hole transport materials. ${ }^{66,67}$ Otherwise, 
materials with an ultra-high electron affinity, such as molybdenum oxide $\left(\mathrm{MoO}_{3}\right), 2,3,5,6-$ tetrafluoro-7,7,8,8-tetracyanoquinodimethane (F4TCNQ) and 1,4,5,8,9,11hexaazatriphenylene hexacarbonitrile (HATCN) used as an interlayer can meet the requirement for higher HOMO, although their working principle is different. ${ }^{68-70}$ For the cathode side, researchers are engaged in finding materials that could create interface dipoles to reduce the work function of the stable metal electrodes. Inorganic salts, such as $\mathrm{LiF}, \mathrm{CaF}_{2}$ and $\mathrm{Cs}_{2} \mathrm{CO}_{3}$ still dominate, although they are not perfect in terms of device stability. ${ }^{21,71}$ In an inverted bottom-emitting OLED, the performance of these inorganic salts used as the interlayer of bottom cathode, such as transparent metal oxides ITO and $\mathrm{ZnO}$ is unacceptable. Instead, polymeric dipole layers, such as PEI, polyethylenimine ethoxylated (PEIE) and small-molecule electron transport materials (ETMs), such as BPhen, bathocuproine (BCP) used either originally or $n$-doped on top of the cathode show excellent electron injection properties. ${ }^{26,72}$ The combinations of $\mathrm{Ag} / \mathrm{MoO}_{3}$ or $\mathrm{Al} / \mathrm{MoO}_{3}$ are usually used as the top anode in these devices. OLEDs with external quantum efficiency (EQE) as high as $30 \%$ have been developed with the optimization of energy levels between the neighboring layers and the synthesis of new types of emitters, such as phosphorescent and thermally activated delayed fluorescent materials. ${ }^{73}$

(a)

\section{Cathode}

ETL

Emitting layer

HTL

Transparent anode

(b)

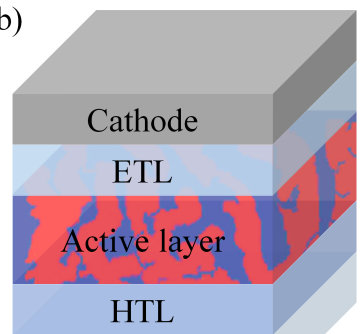

Transparent anode
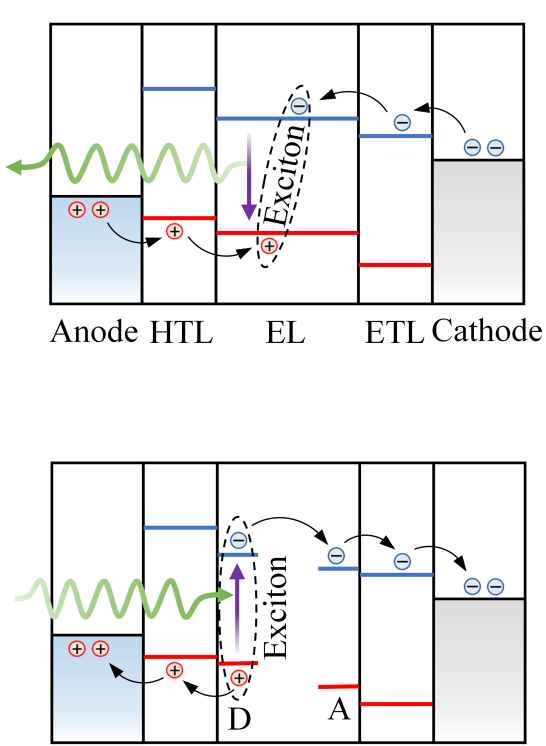

Anode HTL Active ETL Cathode layer

Figure 3.1 Structures (left panel) and energy level diagrams (right panel) of the traditional (a) OLED and (b) OPV devices. 
Similar to OLEDs, typical OPVs also have an anode-OS-cathode sandwich structure, as shown in Figure 3.1b. Under illumination, photons are absorbed by the active layer to generate excitons. The later will be dissociated into free charges, which are subsequently transported to the electrodes. In a donor-acceptor (D-A) heterojunction, exciton dissociation occurs at the $\mathrm{D}-\mathrm{A}$ interface, where holes are formed and transported on the HOMOs of donors, and electrons are formed and transported on the LUMOs of acceptors. They are finally collected respectively at the anode and cathode to form a continuous photocurrent. Due to the converse photophysical process in OPVs, there are several differences in the design of the devices. One is that to improve the photon utilization, donors and acceptors used in OPVs usually feature narrower optical band gaps, which makes the energy level matching between the electrodes and the organic active layer easier. Therefore, a thin layer of ETM is commonly used as the cathode interlayer for the extraction of electrons in a traditional structure and n-type metal oxides are commonly used in an inverted structure. ${ }^{24,74,75}$ The work function of PEDOT:PSS is also high enough for hole extraction. The other one is that the exciton dissociation usually occurs at the D-A interface both in the bulk and planar heterojunctions, which is different from the charge recombination that occurs only on the host material in the OLEDs with a doped EL. Therefore, the energetics at the D-A interface are critical to achieve a high photon-to-current conversion efficiency. Besides, materials used in high-efficient OPVs are mostly polymers and molecules with high molecular weight to extend the conjugated structure and decrease the optical band gap. They are more suitable for solution process rather than the thermal evaporation method commonly used in OLEDs. The morphology of the prepared films is susceptible to the atmosphere and post-treatment, which may further affect the interface energetics.

\subsection{Energy level alignment}

We have introduced the common interfaces in OLEDs and OPVs above. Understanding their energy level alignment is the basic requirement for predicting and regulating the charge injection/extraction barrier between different layers. On this context, we need to identify the interaction of different interfaces first. According to the interaction strength, the interfacial behaviors can be classified from weak to strong as: i) physisorption without any charge transfer. It is mostly found on the clean metal surface covered by noble gas atoms or saturated hydrocarbons, e.g., Au electrode exposure to air. ii) Physisorption possibly with integer charge transfer through tunneling. This scenario usually occurs when OSs are in contact with organic or passivated metal surfaces. iii) Weak chemisorption possibly with partial charge transfer. It is usually observed when OSs are deposited on the non-reactive clean metal surfaces. iv) Strong chemisorption with (partial) charge transfer via covalent bonding. A typical example is OSs growing on reactive clean metal surfaces. 
v) Strong chemisorption with surface dipole and (partial) charge transfer via covalent bonding of molecules and metal at specific sites. It is often seen for OSs with intrinsic dipole and anchoring group deposited on clean metal surfaces, e.g., self-assembly monolayer (SAM) on metal electrode. ${ }^{30}$

Due to the different interactions, there is no unified model for describing all types of interfaces above. It means that to figure out the interfaces in an optoelectronic device, we may need to use more than one model. Therefore, it is important to correctly distinguish the type of interface and choose the appropriate model. Here we use an atomically clean metal as an example to discuss the common interfaces in the device, as shown in Figure 3.2. The work function $\left(\Phi_{\mathrm{m}}\right)$ of the metal is marked as the energy difference between the Fermi level $\left(\mathrm{E}_{\mathrm{F}}\right)$ and vacuum level (VL) and represents the energy required to take out an electron from the metal to the vacuum level, which has been widely explored for most metals that used as electrode. It is important to note that the work function of a metal depends on the crystalline surface due to the different surface dipoles formed by the tailing of the electron density at different packed faces, as show in Figure 3.3a. ${ }^{76}$ For example, the (110), (100) and (111) single crystals of silver show work functions of $4.14 \mathrm{eV}, 4.22 \mathrm{eV}$ and $4.46 \mathrm{eV}$, respectively. ${ }^{77}$
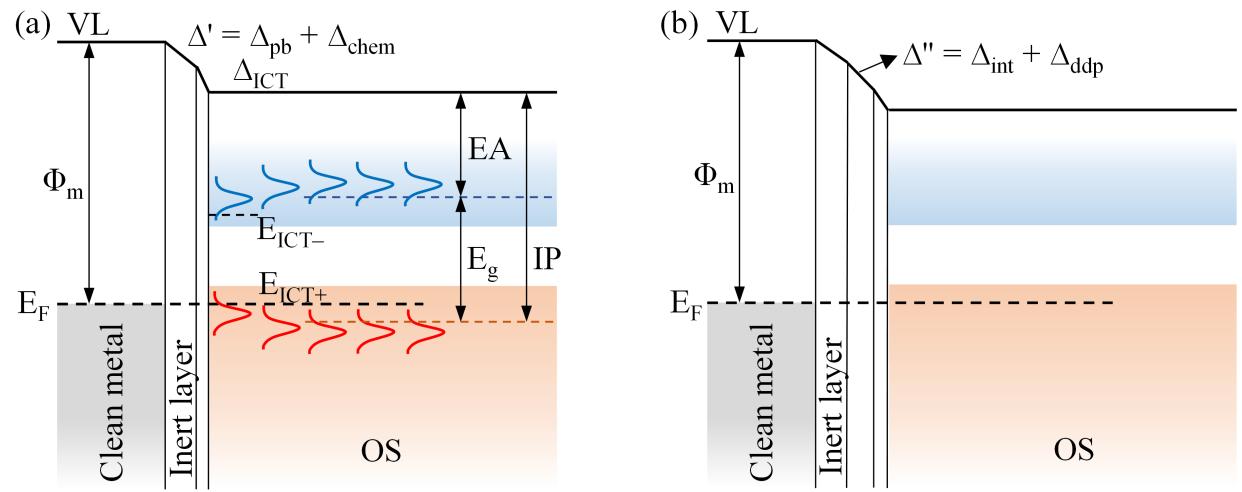

Figure 3.2 Energy level alignment at an OS/clean metal interface. The thin oxide and/or contaminated layer contributes to the interface dipole $\left(\Delta^{\prime}\right)$ through push-back effect $\left(\Delta_{\mathrm{pb}}\right)$ and partial charge transfer $\left(\Delta_{\text {chem }}\right)$. The deposition of OS contributes to the interface dipole (a) only by integer charge transfer $\left(\Delta_{\mathrm{ICT}}\right)$ and (b) by $\Delta_{\mathrm{ICT}}$ together with an additional dipole $\left(\Delta^{\prime \prime}\right)$ composed by the intrinsic dipole moment $\left(\Delta_{\text {int }}\right)$ and double dipole potential $\left(\Delta_{\text {ddp }}\right)$ under certain conditions. Adapted from Ref. 17.

Atomically clean means that there is no oxide or contaminations on the surface of the metal. The preparation and characterization process requires to be carried out in an ultra- 
high vacuum ( $\leq 10^{-9}$ mbar). For the fabrication of electronic devices, the metal electrode is usually exposed to air or inert atmosphere. In the presence of oxygen, an oxide layer will be formed on the surface of reactive metals, which modifies the work function in two approaches. First, the short distance between oxygen atoms and metal surface enables the compression of the electron density tail extended out of the metal surface and forms a pushback dipole $\left(\Delta_{\mathrm{pb}}\right)$ as shown in Figure 3.3b. It reduces the work function in a large extent and has been observed in many cases. For instance, the work function reduction of clean gold upon contacting polymer or contaminants is about $0.7-0.8 \mathrm{eV}$ and clean metals upon noble gas (e.g., Ar, Kr, Xe) absorption is over $1.0 \mathrm{eV} .^{78,79}$

(a)
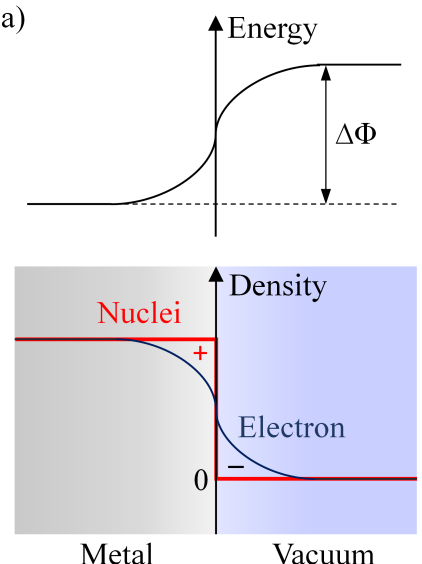

(b)
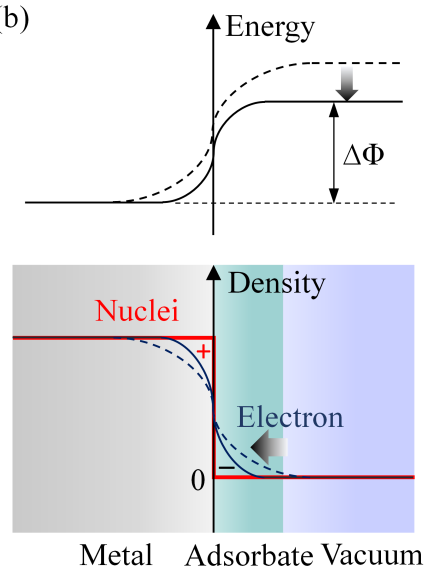

Figure 3.3 (a) A schematic diagram of the distribution of nuclei and electron at a metal surface and the corresponding electrostatic potential energy. (b) The change of charges and energy caused by push-back effect at the metal surface. The adsorbed layer could be an oxide or contaminated layer, such as heteroatoms and small molecules. $\Delta \Phi$ is the surface dipole energy.

Second, the formation of oxygen-metal bond contributes to the modification of the work function through partial charge transfer and the formation of surface dipole $\Delta_{\text {chem }}$. This contribution can be either positive or negative, depending on whether the oxygen atoms are adsorbed on the surface or at the subsurface. ${ }^{80,81}$ The oxide layer on top of the metal electrode will be hydroxyl terminated, followed by a physisorbed layer of water, hydrocarbons and other small molecules when exposure to air, which will form a dipole moment pointing away from the surface and decrease the work function significantly. ${ }^{82}$ In the case of non-reactive metals or the reactive metals in the inert atmosphere, there will be a physisorption of heteroatoms and small molecules directly on the metal surface and the work function is reduced by the push-back effect. Even if the electrode is made in-situ, its 
surface may also be slightly contaminated and/or oxidized due to the higher pressure used during the deposition process $\left(>10^{-7}\right.$ mbar). Therefore, the work function of the metal electrode used in device is usually somewhat different from the corresponding clean metal and depends less on the crystalline surface. Since the push-back effect is present in all systems and always decreases the work function, the work function of electrode is usually lower than the clean metals.

With the deposition of OSs on the oxidized or slightly contaminated metal surface, chemisorption may be still present at the interface via chemical bonding between molecules and the metal surface, such as the sulfur-gold bond observed in some SAM systems. ${ }^{83-86}$ In this case, except for the chemical bond induced dipole $\Delta_{\text {chem }}$ at the OS-metal interface, it will form another dipole $\left(\Delta_{\text {int }}\right)$ in the monolayer when the OS molecules have intrinsic dipole moments and ordered orientation, as shown in Figure 3.2b. If the direction of the intrinsic molecular dipole moments points to the metal surface, the work function will increase, while the dipole moments with opposite direction will cause the work function to decrease. ${ }^{87,88}$ Therefore, the direction of the dipole $\Delta_{\text {int }}$ could be the same as or opposite to that of the dipole $\Delta_{\text {chem }}$, and the work function modification induced by chemisorption could be enhanced or weakened.

Another type of dipole is observed and is hardly involved by the existing systems for the disordered polyelectrolytes and tertiary aliphatic amines, such as poly(4-styrenesulfonic acid) (PSSH), Nafion, and PFN. ${ }^{89}, 90$ From the experimental results, several properties of the dipole can be concluded: i) it occurs on (semi-)conducting substrates and is independent of the substrate work function; ii) the direction depends on the movability of the positive and negative charged species; iii) it is formed at the first layer of the polymer and regardless of thickness. Based on these observations, a "double dipole step" model is proposed to explain the formation mechanism of the dipole. In detail, when the positively and negatively charged species, e.g., cations and anions in the polyelectrolytes and nitrogen nuclei and lone pairs of electrons in the tertiary aliphatic amines, are in contact with the (semi-)conducting substrate, the substrate surface is polarized, forming image charges. Compared with the charged species, their respective image charges formed in the substrate have the same distance from the substrate surface and opposite charges. Therefore, the charged species are attracted by their image charges and move towards the substrate surface, as shown in Figure 3.4 . 

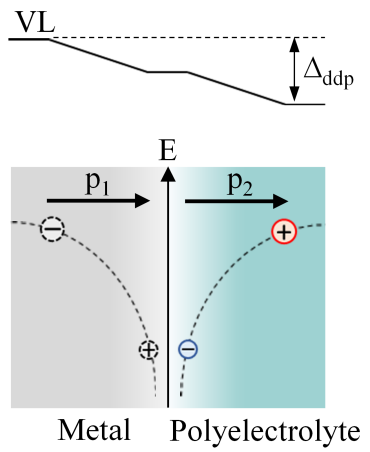
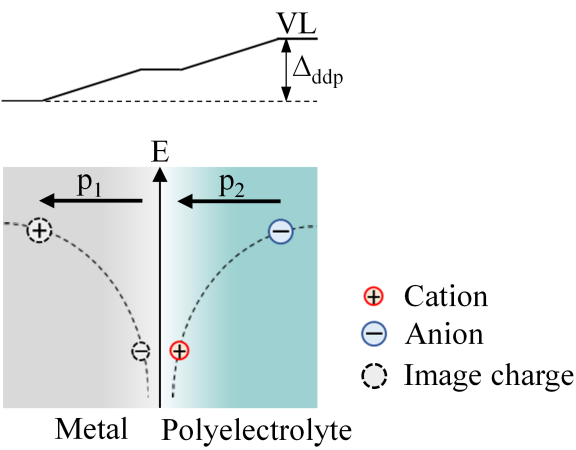

Figure 3.4 Illustrations of the formation of double dipole potential step at the metal/polyelectrolyte interface caused by the size difference between anions and cations.

The equilibrium position of the charged species depends on their size and ability to move in the interlayer, which determines the final size and direction of the dipole. For example, if the negative species is more mobile, it will be closer to the substrate and form a double dipole, $\Delta_{\text {ddp }}$, pointing to the interlayer to downshift the vacuum level. Otherwise, if the positive species is more mobile, the vacuum level will be upshifted, see Figure 3.4. Besides the polyelectrolytes and tertiary aliphatic amines, the double dipole step is also observed in small molecules containing imine $\mathrm{N}$, e.g., pyridine group, and molecularly doped OSs. Especially in the former case, the small-molecule ETMs BPhen and BCP exhibit double dipole potentials as large as $-1.4 \mathrm{eV}$ and $-1.6 \mathrm{eV}$, respectively, ${ }^{91,92}$ making them efficient cathode interlayers in, e.g., OLEDs and OPVs for electron injection/extraction.

We have discussed the energy level alignment of most types of interfaces we mentioned at the beginning of this section. However, a more general interfacial behavior is observed when OSs are in contact with passivated substrates, where there is no push-back effect and chemical interactions due to the electron coupling between the $\pi$-electrons and substrate bands is blocked by the inert layer. It is the second scenario that OSs are weakly physisorbed on the conducting substrates, which are typically formed in, e.g., conjugated polymers spincoated and small molecules spin-coated or sublimated on substrates that are passivated by oxides or residual hydrocarbon contaminants. Here, the polymers and small molecules should be high-quality and undoped to ensure the deficiency of free charges in the film. The large vacuum-level shift ( $\Delta_{\mathrm{ICT}}$ in Figure 3.2) observed at such an interface is attributed to the spontaneous oxidation or reduction of OSs to form polarons at the interface through electron tunnelling. It means integer charge transfer, i.e., one electron at a time, occurs 
between the OSs and substrates under certain conditions, which will be discussed in detail below.

\subsection{Integer charge transfer (ICT) model}

The energy level alignment behavior of the interface mentioned above is well described and explained by the integer charge transfer (ICT) model. ${ }^{30,31,93,94}$ When the work function of the substrate $\left(\Phi_{\text {sub }}\right)$ is higher than the smallest energy required $\left(\mathrm{E}_{\mathrm{ICT}+}\right)$ to take away one electron from the OS near the interface to produce a fully relaxed state, the electron will transfer from the molecule to the substrate and equilibrate until the Fermi level aligned to $\mathrm{E}_{\mathrm{ICT}+}$; when the work function of the substrate is lower than the largest energy gained ( $\left.\mathrm{E}_{\mathrm{ICT}-}\right)$ to add one electron to the OS near the interface to produce a fully relaxed state, the electron will transfer from the substrate to the molecule and equilibrate until the Fermi level aligned to $\mathrm{E}_{\mathrm{ICT}-}$. Due to most $\pi$-conjugated molecules and polymers are soft materials, the fully relaxed state should include both electronic and geometrical relaxation. In addition, the screening effect from the substrate is non-ignorable at the interface, making the ICT states different from the polaronic states in the bulk. Hence, the ICT states usually reside in the forbidden band gap with large offsets from the HOMO and LUMO. According to the ICT model, the relationship between OS-modified work function $\left(\Phi_{\text {sub/org }}\right)$ and $\Phi_{\text {sub }}$ is composed of three regimes. They are:

(i) $\Phi_{\text {sub }}<\mathrm{E}_{\mathrm{ICT}-\text { : }}$ Fermi level pinning to ICT- state via integer electron tunnelling from the substrate to OS, resulting in an upshift of vacuum level $\left(\Delta_{\mathrm{ICT}}\right)$ and constant work function, $\Phi_{\text {sub/org }}=\mathrm{E}_{\mathrm{ICT}-}$, slope $=0$

(ii) $\mathrm{E}_{\mathrm{ICT}-}<\Phi_{\text {sub }}<\mathrm{E}_{\mathrm{ICT}+}$ : Vacuum level alignment, resulting in a substrate-dependent work function without charge transfer, $\Phi_{\text {sub/org }}=\Phi_{\text {sub }}$, slope $=1$

(iii) $\Phi_{\text {sub }}>\mathrm{E}_{\mathrm{ICT}+}$ : Fermi level pinning to ICT+ state via integer electron tunnelling from the OS to substrate, resulting in a downshift of vacuum level and constant work function, $\Phi_{\text {sub/org }}$ $=\mathrm{E}_{\mathrm{ICT}+}$, slope $=0$.

The evolution of the energy level alignment for all three cases are illustrated in Figure 3.5. When OSs are in contact with the substrate, electrons in both (i) and (iii) will spontaneously flow between the substrate and OS, creating dipoles at the interface that upshift or downshift the vacuum level. The electron flow reaches equilibrium when $\Delta_{\text {ICT }}$ is equal to the difference between $\Phi_{\text {sub }}$ and $\mathrm{E}_{\mathrm{ICT}}$, i.e., $\mathrm{E}_{\mathrm{ICT}-}$ in case (i) and $\mathrm{E}_{\mathrm{ICT}+}$ in case (iii). As a result, the work function $\Phi_{\text {sub/org }}$ at equilibrium is equal to the respective EICT. While $_{\text {ICT }}$ for the case (ii), there is no electron flow between the substrate and the OS and no dipole 
formation at the interface. Therefore, this case belongs to the vacuum level alignment and thus $\Phi_{\text {sub/org }}$ is equal to the work function of the substrate $\Phi_{\text {sub }}$.

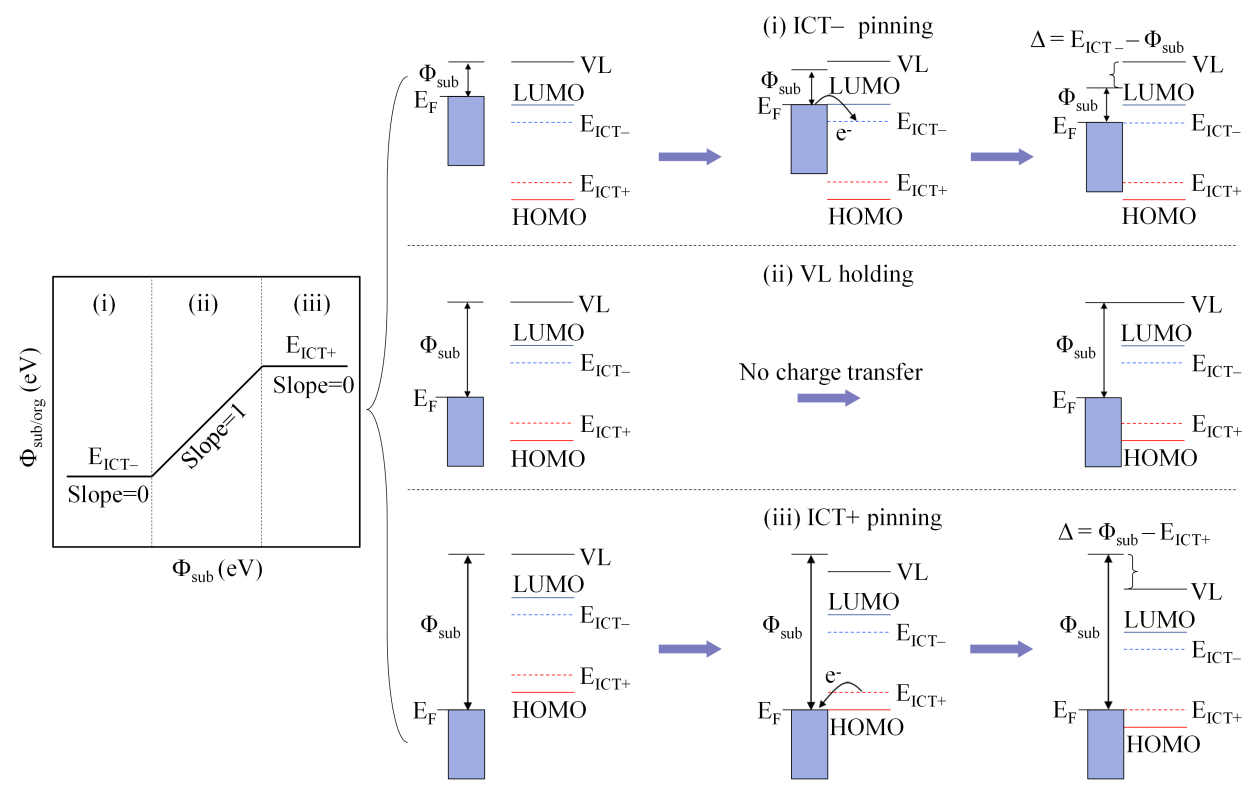

Figure 3.5 A typical ICT curve showing the substrate-dependence of $\Phi_{\text {sub/org }}($ left) and the schematic illustration (right) of the evolution of the energy level alignment corresponding to three regimes described above. Adapted from Ref. 30.

The ICT model provides a guidance for the interface design of the optoelectronic devices and predicts the smallest charge injection barrier at an ohmic contact electrode/OS interface, which is decided by the energy difference between $\mathrm{E}_{\mathrm{ICT}+}\left(\mathrm{E}_{\mathrm{ICT}-}\right)$ and HOMO (LUMO) for hole (electron). To achieve ohmic contact, the work function of the anode should be equal or larger than $\mathrm{E}_{\mathrm{ICT}}+$ and the work function of cathode should be equal or

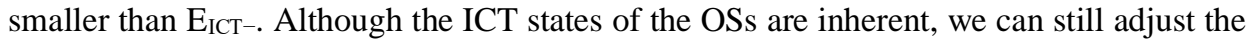
injection barrier by inserting a space layer to weaken the screening from the substrate. As observed by Lindell, L. et al, the $\mathrm{E}_{\mathrm{ICT}-}$ and electron injection barrier are reduced by $0.3 \mathrm{eV}$ by inserting a thin layer of SAMs. ${ }^{95}$

In addition, it has been demonstrated that the ICT model can be modified by the double dipole potential step in the cases of polyelectrolytes, tertiary aliphatic amines, smallmolecule ETMs and molecular doped OSs. ${ }^{90,91,96-98}$ The ICT curve shifts together by $\Delta_{\text {ddp }}$ in these systems, as shown in Figure 3.6. In the case of small molecule BPhen, the nitrogen atoms on the pyridine rings feature free electron lone pairs in $\mathrm{sp}^{2}$ orbitals that overlap little 
with the aromatic $\pi$-system. The lone pairs and nitrogen nuclei compose the negative and positive species. When molecules are deposited onto the substrate, lone pairs in contact with the substrate surface contribute mainly to the formation of double dipole potential, while lone pairs away from the surface are negligible due to the weak polarization to the substrate. As a result, the double dipole moments point away from the substrate, inducing a downshift of vacuum level across the three regimes of the ICT model. The dipole is increased when the film is n-doped, as expected from the model as we introduce additional charged species in the interface layer.

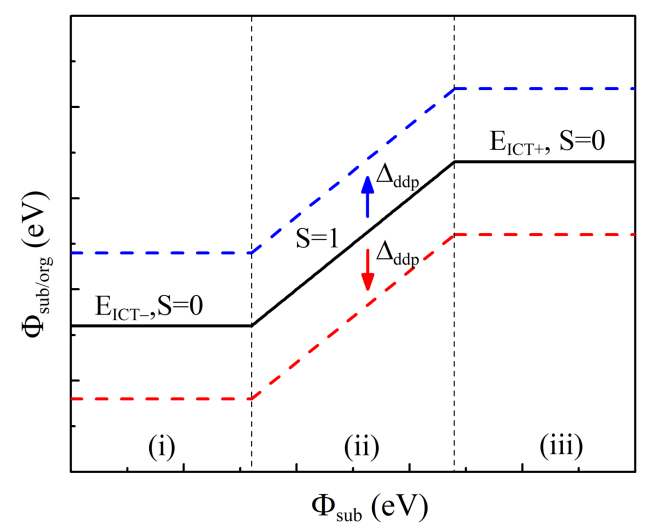

Figure 3.6 ICT curve shift in the double dipole potential step $\left(\Delta_{\mathrm{ddp}}\right)$ modified ICT model. 


\section{Chapter 4 Chemical doping in organic semiconductors}

Pure OSs feature negligible intrinsic charge carriers that limits their applications in many areas. Chemical doping is one of the most efficient methods to introduce and control extra charges in OSs and is widely used in such as OLEDs, OPVs, OFETs and conducting fibers. There are various types of dopants used in OSs, such as metals, molecules, polymers, and ions, etc. Small inorganic molecules iodine and $\mathrm{FeCl}_{3}$ are mostly used in the early chemical doping of conjugated polymers. ${ }^{99,100}$ Neutral organic molecules with either high electron affinity (EA) or low ionization potential (IP) can also provide strong electron accepting or donating ability. Polymers such as polystyrene sulphonic acid (PSS) is known for using as the dopant of PEDOT. ${ }^{101}$ Other dopants such as Lewis acids and bases have also been widely used and explored in depth. ${ }^{102}$

Although these dopants can eventually bring charges to the OS matrix, their working principles and interaction with OS are different. Thereinto, Brønsted acids or bases are considered to react with OSs via protonation, while neutral molecules dope OSs through charge transfer with varying degrees of chemical interaction. Lewis acids and bases induce charges through coordination effects with the OSs or through an anion assisted electron transfer. All types of dopants can provide efficient doping and are widely studied. Here, we will be focusing specifically the charge transfer scenario, which is associated with our studies.

\subsection{Doping fundamentals}

Chemical doping through charge transfer is borrowed from the inorganic semiconductors, which is usually doped by substituting partial atoms or inserting interstitial atoms in the crystal matrix. Electron donor impurities creates new occupied states near the conduction band edge (i.e., n-type doping) and electron acceptor impurities creates new unoccupied states near the valence band edge (i.e., p-type doping). Accordingly, the Fermi level in the band gap will also shift towards the conduction band or valence band. The case of OS doping is similar but not exactly the same due to the narrower bandwidth in OSs, which means the electronic structures of organic molecules or polymers are more localized. Instead, the dopant molecules/atoms either add electrons to the LUMO or remove electrons from the HOMO of the OS. According to the interactions between dopant molecules and OSs, the doping process is understood as either ground-state integer charge transfer or 
hybridization of the electronic wave functions between the host OS and dopant, corresponding to the formation of ion-pairs and charge-transfer complexes, respectively.

\subsubsection{Ion-pair formation}

(a) n-type doping

LUMO

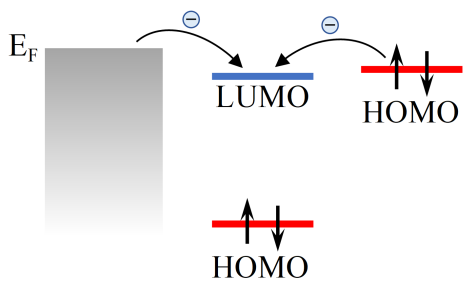

Metal/inorganic OS Neutral organic dopants dopants

(c)

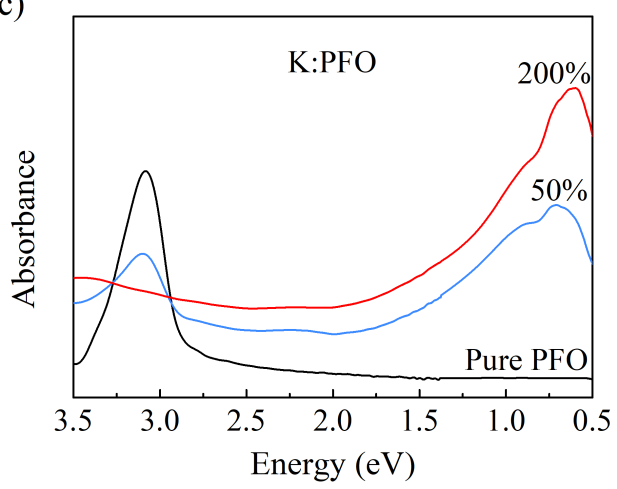

(b) p-type doping

\section{$\overline{\text { LUMO }}$}

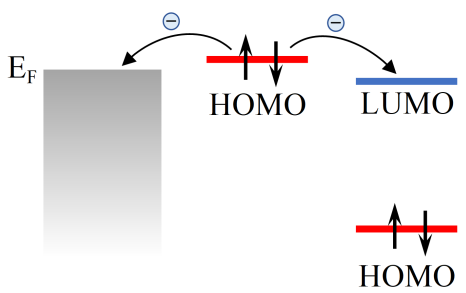

Metal/inorganic OS Neutral organic dopants dopants

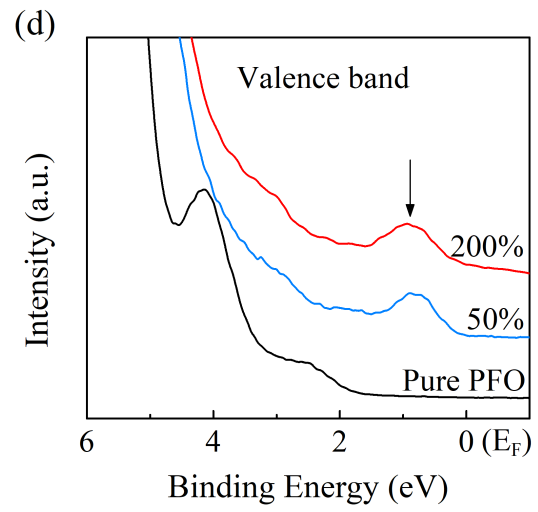

Figure 4.1 (a) Schematic of integer charge transfer in n-type (left) and p-type (right) doping. (b) UV-vis-NIR absorptions (left) and valence band of UPS spectra (right) of PFO films doped with potassium.

In typically chemical doping, integer charge transfer is commonly thought to occur, leading to an electron and hole localized respectively on the dopant and host OS, as shown in Figure 4.1a,b. In such scenarios, each dopant molecule is completely ionized and transfers at least one charge carrier to the OS matrix, resulting in the formation of a polaron or bipolaron. ${ }^{103}$ It is mostly found in the conjugated polymers when doped with strong reductants or oxidants, e.g., alkali metals, inorganic molecules, or some organic molecules and has been evidenced with the spectroscopical fingerprint of the polaron. For example, in 
the potassium-doped PFO, the characteristic UV-vis-NIR absorption of negative PFO polarons and new polaronic states near the Fermi level in the UPS spectrum are observed, as presented in Figure 4.1c,d. The absorption spectra of the potassium-doped film are consistent with those measured in the electrochemically reduced PFO. ${ }^{104}$ Other examples such as the most widely studied polymer poly(3-hexylthiophene) (P3HT), when it is pdoped by the small molecule F4TCNQ, the optical absorptions of both positive and negative polarons as well as the shift of F4TCNQ cyano-vibrations in FTIR spectrum are observed. ${ }^{105}$ However, researchers also found that although all F4TCNQ dopants undergo integer charge transfer with the polymer, only $5 \%$ positive charges on the polymer are free to move and contribute to the conductivity, while most transferred charges are still bound Coulombically together as electron-hole pairs. ${ }^{106}$

One generally accepted prerequisite for the ion-pair formation is the IP (EA) of the organic dopant (or Fermi level of metal/inorganic dopant) should be equal to or lower (higher) than the EA (IP) of the OS in n-type (p-type) doping. As exemplified by a series of dodecaborane derivatives (DDBs) doped P3HT, the absorption signature of P3HT polaron increases as the EA of the dopants increases. ${ }^{107}$ And when the EA of the dopant is smaller than the IP of P3HT, no polaron absorption is observed. However, that is actually not how it is proved, and this prerequisite contradicts with many other experimental observations. ${ }^{108-110}$ The difference between the IP of the donor and the EA of the acceptor accounts for the free energy of electron transfer between two separate molecules. However, in the well mixed doping system, the electrostatic interaction between the donor and acceptor needs to be considered, which will stabilize the ion pairs after electron transfer. ${ }^{111}$ The energy of the ion pair in a doping system can be corrected as the following equation: ${ }^{19}$

$$
E_{\delta}=(I P-E A) \delta-k \frac{e^{2}}{r} \delta^{2}
$$

where $\delta$ represents the degree of charge transfer, $r$ stands for the distance between the positive and negative charges, $k$ and $e$ are the Coulomb's constant and the electric charge, respectively. Except for the energy difference between IP and EA, the energy $\mathrm{E}_{\delta}$ is also dependent on the distance $r$, which is further influenced by the doping concentration, the arrangement of the OS matrix and the distribution of the dopant. In addition, the values IP and EA in the mixed system change significantly with the doping concentration induced by the electrostatic interaction and polarization effect. ${ }^{57}$

\subsubsection{Charge-transfer complex formation}


(a) n-type doping

LUMO
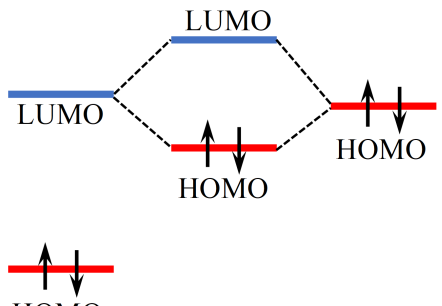

HOMO

Neutral OS CPX Neutral dopant

(c)

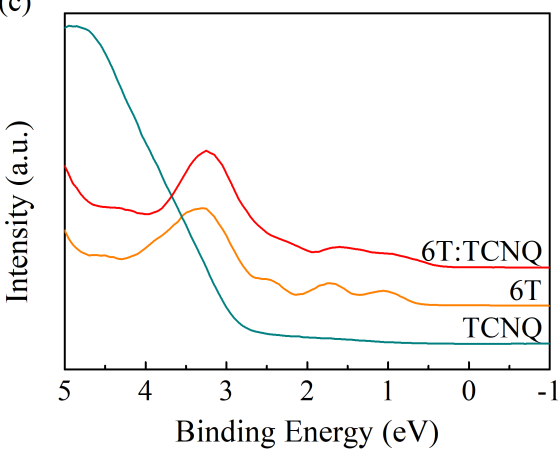

(b) p-type doping

$$
\text { LUMO }
$$
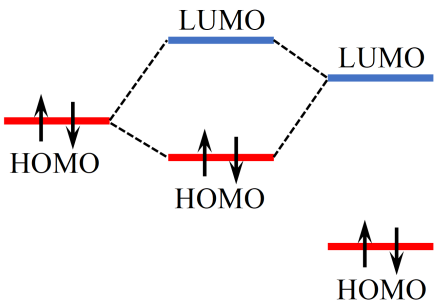

Neutral OS

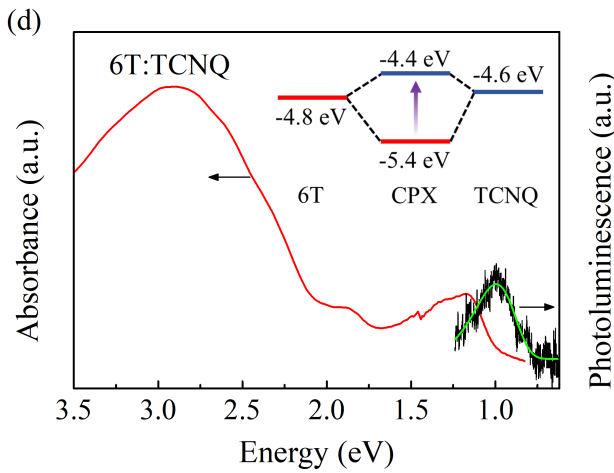

Figure 4.2 The formation of ground-state charge-transfer complex (CPX) in (a) n-type and (b) p-type doping. (c) Valence band structures of 6T, TCNQ and their mixture. (d) UV-visNIR absorption and photoluminescence of 6T:TCNQ mixture.

The ground-state charge-transfer complex (CPX) will be formed when partial charge transfer $(\delta<1)$ occurs during the doping process. Different from the integer charge transfer, the formation of CTC is less dependent on the energy difference between the IP of donor and the EA of acceptor, and has been found even when the IP of donor is significantly larger than the EA of acceptor, such as in the active layer of the organic solar cells employing DA bulk heterojunctions. ${ }^{112,113}$ The overlap of $\pi$-orbitals between two adjacent donor and acceptor molecules induces the formation of supramolecular hybrid orbitals. In this context, two new CPX states are formed by the linear combination between the LUMO (HOMO) of the host OS and the HOMO (LUMO) of the dopant in n-type (p-type) doping, as shown in Figure 4.2. Thereinto, the lower-energy bonding level will be occupied by two electrons from the HOMO of the parent donor and becomes the new HOMO of the CPX, while the higher-energy antibonding level is empty and becomes the LUMO of CPX. The distribution 
of the HOMO and LUMO of the CPX on the two parent molecules is directly related to the degree of the charge transfer $(\delta)$. The energy of the HOMO and LUMO can be derived from the equation:

$$
E_{C P X, H / L}=\frac{H_{D}+L_{A}}{2} \pm \sqrt{\left(H_{D}-L_{A}\right)^{2}+4 \beta^{2}}
$$

where $\mathrm{H}_{\mathrm{D}}$ and $\mathrm{L}_{\mathrm{A}}$ are the parent HOMO and LUMO, $\beta$ is the resonance integral. Therefore, their values are mainly determined by the parent HOMO and LUMO and is independent of the HOMO is higher or lower than the LUMO. The HOMO-LUMO energy gap of the CPX is from a few tenths $\mathrm{eV}$ to $>1 \mathrm{eV}$, e.g., $0.9 \mathrm{eV}$ for quaterthiophene (4T):F4TCNQ derived from the UPS and IPES spectra. ${ }^{105}$

Different from the ion-pair formation, the CPX is charge-neutral, and no new gap state appears near the Fermi level, which has been evidenced by UPS characterizations. It is also difficult to detect the HOMO feature of the CPX by UPS because in most cases the proportion of CPX is only a few percent and the new state will be submerged by the valence features of the host OS. As shown in Figure 4.2c, the valence features of the $\alpha$-sexithiophene (6T) show little change after being doped by 7,7,8,8-tetracyanoquinodimethane (TCNQ). However, a new sub-gap absorption arising from the transition between the new HOMO and LUMO of the CPX is observed via UV-vis-NIR spectroscopy, where the optical band gap of around $1.0 \mathrm{eV}$ is derived, as shown in Figure 4.2d. Similar results have been reported for the 4T:F4TCNQ doping system. ${ }^{105}$ By excitation with a $900 \mathrm{~nm}$ photon source, a weak near-infrared emission is detected around $1 \mathrm{eV}$.

One of the most studied class of CPXs is the molecular D-A cocrystals, where the DA pairs accumulate regularly in a specific stacking mode through electrostatic interactions. ${ }^{14,115}$ Due to the presence of both donor and acceptor molecules, many D-A cocrystals exhibit ambipolar transport properties, which makes it possible to fabricate ambipolar field-effect transistor (FETs). ${ }^{116,117}$ The stacking mode of the cocrystal depends on the mixing ratio and preparation method, which further affects the charge transport properties. Figure 4.3 shows the scanning electron microscope (SEM) and the UV-vis-NIR absorption of $4 \mathrm{~T}$ :TCNQ cocrystal with a mixing ratio of $1: 1$. The crystals are rod-like with length of several micrometers. Compared to the amorphous blending film, the cocrystal shows a much stronger CPX absorption signature and the optical band gap shows a slight decrease. It indicates that the regular arrangement of the D-A molecule in the cocrystal promotes the CPX formation, whereas most molecules are unable to form the CPX due to the inhomogeneous mixing in the amorphous film. 
(a)

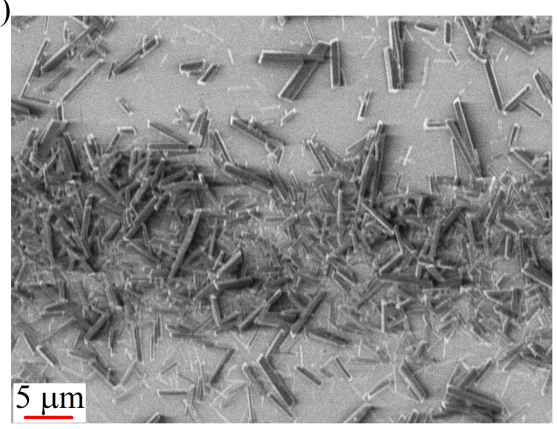

(b)

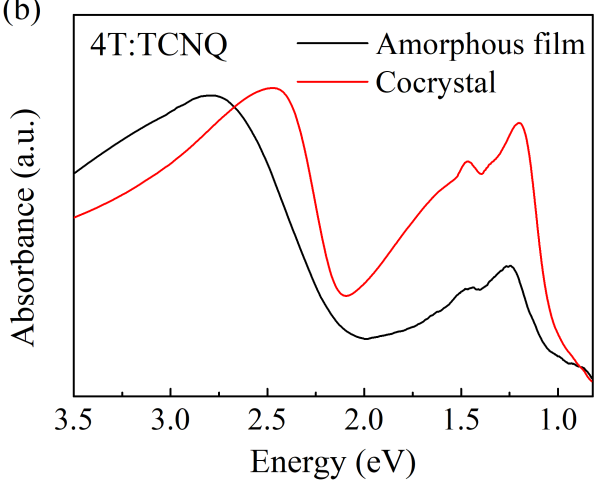

Figure 4.3 (a) SEM of 4T:TCNQ cocrystal prepared by drop-casting method. (b) Comparison of UV-vis-NIR absorption spectra between the amorphous 4T:TCNQ blending film and cocrystal.

\subsubsection{Dedoping}

Dedoping process is to remove the extra charges produced by doping and recover OS molecules to a neutral state. This is based on the fact that many redox reactions in OSs are reversible. Factors associated with the dedoping process include heat, solvents, and chemical reagents. In some doping systems, the dopants can be removed by thermal sublimation, leaving the neutral OS matrix. This is usually found when the dopants have high vapor pressure, such as iodine and oxygen. It has been proved by photoelectron spectroscopy that fullerene PCBM doped with $\mathrm{O}_{2}$ is reversible under heat treatment. ${ }^{97}$ The solvent can cause dedoping by shifting the equilibrium of the doping reaction to the neutral state of the host OS. It is achieved by exposing the doped film to an orthogonal solvent which has poor solubility for the host OS but good solubility for the dopant. An example is the recovery of the UV-vis spectrum when the P3HT:F4TCNQ blend film is immersed in the solvent acetonitrile. ${ }^{118}$ The absorption signatures of the F4TCNQ anion at around 1.5 $\mathrm{eV}$ decline significantly upon the solvent treatment. However, it can only obtain partial dedoping via this method and will stop at a new equilibrium.

In order to achieve complete dedoping, adding competitive donor (acceptor) that is easier oxidized (reduced) than the host OS in p-type (n-type) doping is an effective approach. The competitive donor molecules will react with the charged dopant and donate electrons to the host OS. The production of this process is stable enough to deactivate the dopants. In the above example, by adding the electron donor molecule diethylamine (DEA) into the acetonitrile, the absorption signatures of the charged species disappear completely and are replaced by the absorption of the neutral P3HT. In the n-type doping, the acceptor molecules 
will react with the charged dopant and accept the electrons from the host OS. A prominent example of the competitive acceptor is oxygen. As shown in the following Figure 4.4, the dedoping of potassium-BBL via air exposure is demonstrated both by UV-vis-NIR and UPS spectroscopy. When exposed to air, the absorption signatures of the BBL polaron at $1.5 \mathrm{eV}$ and $0.5 \mathrm{eV}$ disappear, accompanied by the recovery of the neutral BBL absorption. Also, the gap state near the Fermi level in the UPS spectrum disappears completely upon air exposure. Here, the oxygen is the electron acceptor and reacts with the alkali metals to form metal oxides, resulting in the dedoping of the host OS.

(a)

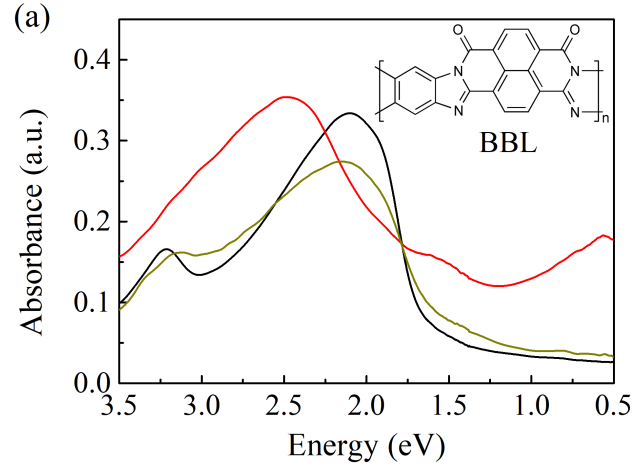

(b)

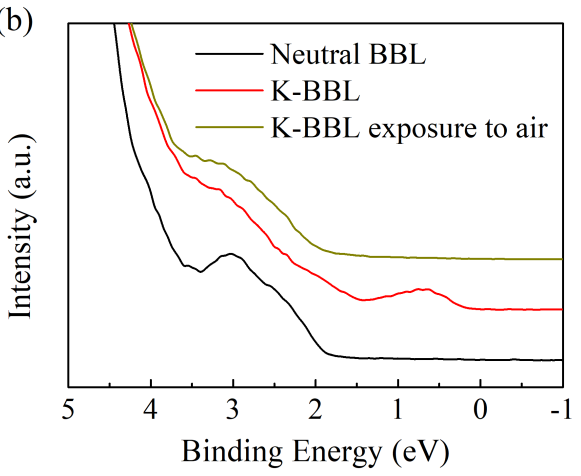

Figure 4.4 (a) UV-vis-NIR and (b) UPS measurements of the potassium-doped BBL before and after exposure to air. The neutral BBL is also included as a reference.

\subsection{Dopants}

To achieve efficient doping, early studies usually use the strong reductants, e.g., alkali metals $\mathrm{Na}, \mathrm{K}, \mathrm{Cs}, \mathrm{Li}$ as n-type dopants and strong oxidants, e.g., halogens $\mathrm{Cl}_{2}, \mathrm{Br}_{2}, \mathrm{I}_{2}$ and salt $\mathrm{FeCl}_{3}$ as p-type dopants. ${ }^{1{ }^{19-121}}$ Due to the extremely low ionization potential and high diffusivity, most OSs can be n-doped by alkali metals via integer charge transfer. The UPS detection of the gap states in the polaron is mainly based on the alkali metal doping. ${ }^{121-125}$ But one thing to note is that they are very sensitive to air, the doping process and the succeeding characterizations need to be carried out in ultra-high vacuum. Some air-stable alkali salts, e.g., $\mathrm{Cs}_{2} \mathrm{CO}_{3}, \mathrm{Rb}_{2} \mathrm{CO}_{3}, \mathrm{Li}_{2} \mathrm{CO}_{3}$ and $\mathrm{CH}_{3} \mathrm{COONa}$ are usually used as substitutes in devices to achieve efficient n-doping by tuning the energy levels and increasing conductivity. Halogens not only exhibit strong oxidizing properties, but are also volatile (including $\mathrm{I}_{2}$ ), which allows them to diffuse into the OS films as a gas at room temperature. By gently heating, the halogens can be easily removed to dedope the film. However, the strong volatility also brings a big problem, that is, the doping ratio is difficult to control. Transition metal oxides, such as $\mathrm{MoO}_{3}, \mathrm{WO}_{3}$, and $\mathrm{V}_{2} \mathrm{O}_{5}$ are widely used in electronic 
devices as p-type dopants due to their high EAs. ${ }^{126}$ In the following subsections, the commonly used n-type and p-type organic-based dopants are introduced briefly.

\subsection{1 n-type molecular dopants}

Except for the alkali metals and their salts, a wide variety of n-type organic and organometallic compounds with low IP have been developed, which are listed in Figure 4.5. Bis(ethylenedithio)tetrathiafulvalene (BEDT-TTF) is the first n-type organic dopants used in the OS doping by Nollau et al. ${ }^{127}$ After that, other neutral organic compounds, such as tetrakis(dimethylamino)ethylene (TDAE) and 1,8-Diazabicyclo[5.4.0]undec-7-ene (DBU) are explored as n-type dopants for conjugated polymers or molecules with relatively higher EA, which can improve the conductivity significantly. ${ }^{128-130}$ Some transition metal complexes, such as cobaltocene and rhodocene, are inclined to lose one electron to form stable 18-electron cations, which endows them strong n-type doping abilities. For instance, decamethylcobaltocene $\left(\mathrm{Co}\left(\eta_{5}-\mathrm{C}_{5} \mathrm{Me}_{5}\right)_{2}\right)$ shows an IP of $-3.3 \mathrm{eV}$, which is low enough to transfer electrons to the LUMO of a large proportion of OSs. When doping copper phthalocyanine $(\mathrm{CuPc})$, whose EA is about $-3.5 \mathrm{eV}$, the current density is increased $10^{6}-$ fold by reducing the electron injection barrier and enhancing conductivity in the $\mathrm{Au} / \mathrm{CuPc} / \mathrm{Au}$ device. ${ }^{131}$ The complexes $\mathrm{M}_{2}(\mathrm{hpp})_{4}$, where $\mathrm{M}$ is metal, e.g., Mo, $\mathrm{W}$ or $\mathrm{Cr}$ and hpp represents the anion of 1,3,4,6,7,8-hexahydro- $2 H$-pyrimido[1,2-a]pyrimidine, are also strong n-type dopants with ultra-low IP. The conductivity of fullerene $\mathrm{C}_{60}$ doped by such molecules can reach to $4 \mathrm{~S} \mathrm{~cm}^{-1}{ }^{132}$

However, similar to alkali metals, these compounds are also unstable in air due to the low IP. One alternative solution is using air-stable precursors, such as transition metal complex dimers, which can be converted into active intermediates after thermal activation or photoactivation. This type of dopants provides a promising approach for stabilizing ultralow IP dopants and reducing the requirement of the doping process. The precursor (pentamethylcyclopentadienyl)(1,3,5-trimethylbenzene)ruthenium dimer ([RuCp*Mes $]_{2}$ ), which have been used in the doping of pentacene and $\mathrm{C}_{60},{ }^{133,134}$ is found to be able to ndope the ETM phenyldi(pyren-2-yl)phosphine oxide $\left(\mathrm{POPy}_{2}\right)(\mathrm{EA} \sim-2.2 \mathrm{eV})$ after UV activation and is air-stable without the irradiation. ${ }^{135}$ The other type of precursor is the organic hydrides, such as the benzimidazole derivatives N-DMBI and 4-(4methoxyphenyl)-1-methyl-2,6-diphenyl-1,4 dihydropyridine (DPDHP), which have also been evidenced to be efficient n-type dopants. ${ }^{136-138}$ 
<smiles>C1CSC2SC(=C3SC4SCCSC4S3)SC2S1</smiles>

BEDT-TTF

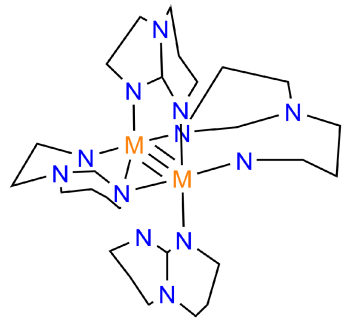

$\mathrm{M}_{2}(\mathrm{hpp})_{4}$

$\mathrm{M}=\mathrm{Mo}, \mathrm{W}, \mathrm{Cr}$<smiles>CN(C)c1ccc(C2N(C)c3ccccc3N2C)cc1</smiles>

N-DMBI<smiles>CN(C)C(=C(N(C)C)N(C)C)N(C)C</smiles>

TDAE
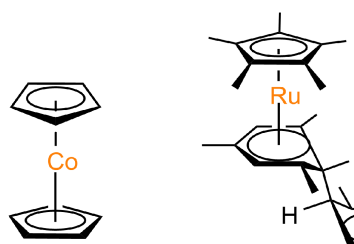

DBU

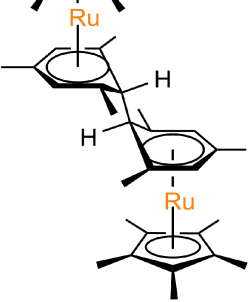

$\left[\mathrm{RuCp}{ }^{*} \mathrm{Mes}\right]_{2}$<smiles>COc1ccc(C2C=C(c3ccccc3)N(C)C(c3ccccc3)=C2)cc1</smiles>

DPDHP
$\mathrm{HOMO} / \mathrm{WF}(\mathrm{eV})$<smiles>CC(C)(C)OC(=O)C(C)(C)[AlH2]</smiles>

$\operatorname{Co}\left(\eta_{5}-\mathrm{C}_{5} \mathrm{Me}_{5}\right)_{2}$ $\mathrm{W}_{2}(\mathrm{hpp})_{4}--3.5$

BEDT-TTF $\left.\mathrm{Cr}_{2}(\mathrm{hpp})_{4}\right]^{-4.7}$

Figure 4.5 Molecular structure of several commonly used n-type dopants. The HOMO or work function of partial molecules comes from various measurements and different literatures.

\subsection{2 p-type molecular dopants}

Unlike n-type dopants, p-type dopants with high EA usually show good stability in air. Except for the halogens and inorganic salts mentioned above, a large number of p-type organic molecules have been designed by introducing electron-withdrawing groups, e.g., fluorine atom and cyan group. In particular, the prototypical small molecule TCNQ has been widely studied in the formation of CPX due to its moderate oxidability (EA $=-4.6$ $\mathrm{eV}$ ). In order to achieve efficient doping of most OS molecules, the EA of dopants should be higher than $-5.0 \mathrm{eV}$ to accept electrons from the host OS via integer charge transfer. Figure 4.6 shows the chemical structure of commonly used p-type dopants as well as their EAs. The fully fluorinated derivative of TCNQ, F4TCNQ, is one of the most studied p-type dopants with an EA of $-5.24 \mathrm{eV}$ and has been widely used to dope conjugated polymers. In particular, the doping of P3HT by F4TCNQ has become a prototype for exploring the doping mechanism and the electronic structures of positive polarons. ${ }^{139-142}$ Some other analogues, such as hexafluorotetracyanonaphthoquinodimethane (F6TCNNQ) and 3,5difluoro-2,5,7,7,8,8-hexacyanoquinodimethane (F2HCNQ) show even higher EA and 
stronger oxidability than F4TCNQ. 1,4,5,8,9,11-hexaazatriphenylenehexacarbonitrile (HATCN) with a larger $\pi$-conjugated structure also shows a high EA of $-4.7 \mathrm{eV}$ and is usually used as the hole injection layer in OLEDs to increase the work function of anode. Another commonly used p-type dopant is the organometallic complex Mo(tfd) $)_{3}$, consisting of three dithiolene ligands substituted by trifluoromethyl. It shows a good stability and high doping efficiency with an EA of $-5.6 \mathrm{eV} .{ }^{143,144}$

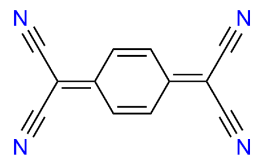

TCNQ

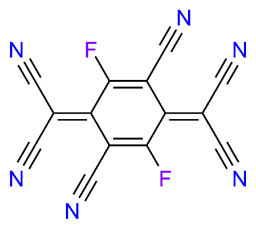

F2HCNQ

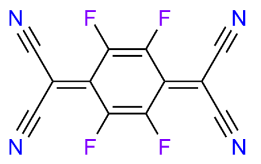

F4TCNQ

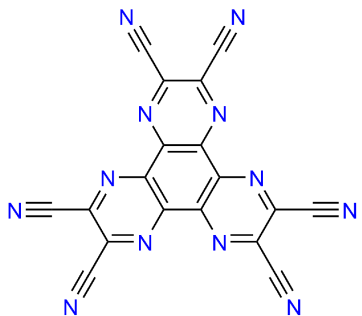

HATCN

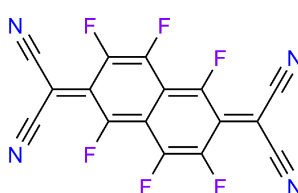

F6TCNNQ<smiles>[R]C1=C([CH])S[N+]2(S1)SC([R])=C(C(F)(F)F)S2</smiles>

$\mathrm{Mo}(\mathrm{tfd})_{3}$

LUMO/WF (eV)

Figure 4.6 The molecular structure of commonly used p-type dopants (left) and their LUMO or work function (right). ${ }^{19,126}$ It shows the work functions for three transition metal oxides and LUMO values for other organic or organometallic molecules.

\subsection{Applications of chemical doping in optoelectronic devices}

Charge transport in thin films is a crucial process in the operation of the optoelectronic devices, e.g., OLEDs and OPVs. Due to the inherent low mobility, the carrier transport in the undoped OS will cause additional energy loss, such as an increase in the operating voltage of OLEDs or a decrease in the charge carriers collected in OPVs. In order to achieve a low ohmic loss and efficient transport, both matched energy levels and high conductivity are required for the charge transport layer. Also, the efficient charge injection/extraction from the electrode to the OS needs a low energetic barrier. In view of these issues, $\mathrm{p}$ - or ntype chemical doping is employed in the charge transport and injection/extraction layers to improve the carrier mobility, tune the energy levels, and minimize the injection barriers. In addition, doping is also commonly found in the host-guest emitting layer of OLEDs and the bulk heterojunction active layer of OPVs. 


\subsubsection{Doped layers in OLEDs}

We have introduced the basic structure and the working principle of a traditional OLED device in the last chapter. Due to the weak interactions between the emitter and the host molecules in the emitting layer, there is no ground-state charge transfer. Hence, we only focus on the chemical doping in other layers here. Charge injection from the metallic electrodes to the organic transport layer is a critical step to achieve a high efficiency due to the work function of the electrode usually does not match the transport energy levels of the OS. Introducing interlayers as the injection layer is an effective way to reduce the injection barrier. However, sometimes the work function modified by the pure molecules cannot reach the required value, especially in OLEDs where large band-gap OSs are usually applied. Hence, the interlayers usually use the p- or n-typed doped molecules. The effect of doping on the injection behavior has been widely investigated by UPS and current-voltage characterization. An example for the n-type doped injection layer is BPhen: $\mathrm{Cs}_{2} \mathrm{CO}_{3}$, which is used to inject electrons in the inverted OLED. ${ }^{49}$ As shown in Figure $4.7 \mathrm{a}$, the work function of BPhen doped with $10 \% \mathrm{Cs}_{2} \mathrm{CO}_{3}$ is $0.2 \mathrm{eV}$ lower than that of pure BPhen when deposited on $\mathrm{ZnO}$, which means that the electron injection barrier (i.e., from Fermi level to the LUMO of BPhen) is reduced by $0.2 \mathrm{eV}$. As a result, the current density is increased significantly by using the doped injection layer in the electron-only device (see Figure 4.7b). Not only $\mathrm{Cs}_{2} \mathrm{CO}_{3}$, many other n-dopants such as $\mathrm{Rb}_{2} \mathrm{CO}_{3}, \mathrm{Li}_{2} \mathrm{CO}_{3}$ and $\mathrm{Li}$ are also usually used in the doped electron injection layer of OLED. ${ }^{145-148}$
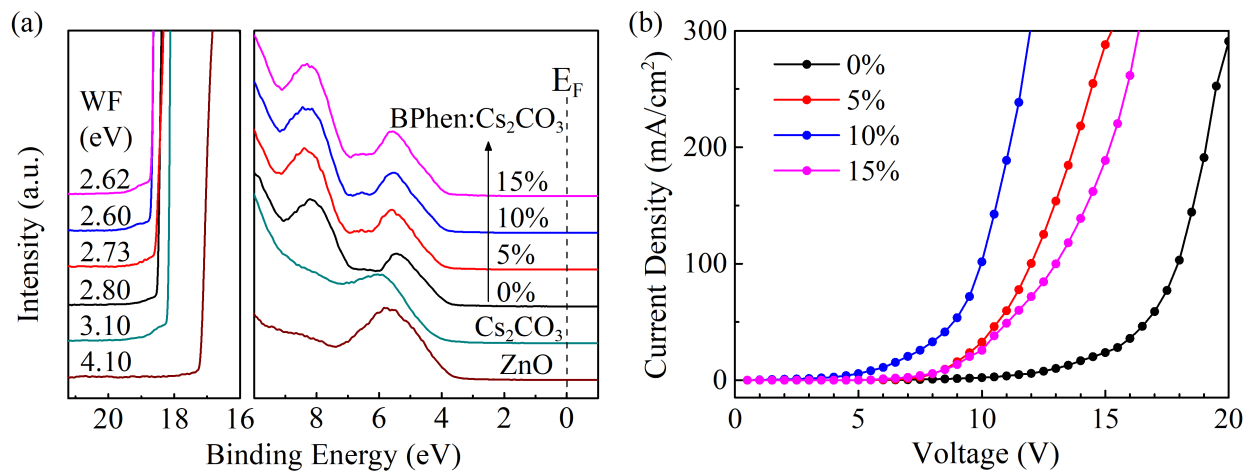

Figure 4.7 (a) UPS spectra of BPhen, $\mathrm{Cs}_{2} \mathrm{CO}_{3}$ and their doped layers on $\mathrm{ZnO}$. (b) Comparison of current density-voltage curves of electron-only device with the change of $\mathrm{Cs}_{2} \mathrm{CO}_{3}$ doping ratio. The structure of the device is ITO/ZnO $(20 \mathrm{~nm}) / \mathrm{BPhen}: x \mathrm{wt} \% \mathrm{Cs}_{2} \mathrm{CO}_{3}$ $(20 \mathrm{~nm}) / \mathrm{BPhen}(20 \mathrm{~nm}) / 3$,3'-Di(9H-carbazol-9-yl)-biphenyl $(30 \mathrm{~nm}) / \mathrm{BPhen}(30 \mathrm{~nm}) / \mathrm{LiF}$ $(0.9 \mathrm{~nm}) / \mathrm{Al}$. Adapted from Ref. 49. 
The most commonly used p-type dopants in the hole injection layer are $\mathrm{MoO}_{3}, \mathrm{WO}_{3}$ and F4TCNQ. As demonstrated by the F4TCNQ doped zinc phthalocyanine ( $\mathrm{ZnPc}),{ }^{149}$ the hole injection barrier from $\mathrm{Au}$ to the undoped $\mathrm{ZnPc}$ is $0.9 \mathrm{eV}$, and the current density is controlled by the injection-limited current. Upon 0.3\% F4TCNQ doping, obvious band bending occurs at the Au/doped-ZnPc interface with a depletion width of $12.8 \mathrm{~nm}$ and a minimum injection barrier of $0.42 \mathrm{eV}$. The current density increases by about three orders of magnitude and becomes a typical space charge limited current (SCLC). With increasing the doping ratio to $3 \%$, the injection barrier is further reduced to $0.18 \mathrm{eV}$ and the depletion width is just $3.2 \mathrm{~nm}$, which indicates the number of free charges in the OS matrix is increased, resulting in the shift of Fermi level toward the HOMO of ZnPc. Similar results have been also obtained in the $\mathrm{MoO}_{3}$ doped N,N'-Di(naphthalene-1-yl)-N,N'-diphenylbenzidine (NPB), which shows a dramatically decreased injection barrier and increased current density for hole injection from ITO/ $\mathrm{MoO}_{3}$-doped NPB. ${ }^{69,150}$

Doped OS is rarely used as a transport layer directly connected to the emitting layer, because excitons are easily quenched by polarons or trapped by the shallow energy levels of the dopants at the transport layer/EL interface. In addition, the diffusion of some small dopants to the emitting layer and the low glass transition temperature of small dopant molecules will significantly reduce the stability and lifetime of the device. ${ }^{151,152}$ For this reason, a thin blocking layer is employed between the doped transport layer and the emitting layer. Sometimes the doped OS also serves as injection layer. For instance, the device is allowed to operate with an ultra-low voltage by employing ITO/NPB:4\% $\operatorname{ReO}_{3}(80$ $\mathrm{nm}) / \mathrm{NPB}(20 \mathrm{~nm})$ on the anode side. ${ }^{153}$ Doping is also widely used in the charge generation layer (CGL) of the vertical stacked OLEDs, which is learned from the inorganic semiconductor devices. A lot of studies have demonstrated that the OS/OS p-n junction is very useful as the connecting unit in the stacking OLEDs. ${ }^{154-157}$ It will form a space-charge region at the interface of the two doped layers, where the vacuum level downshifts largely on the n-doped side, as shown in Figure 4.8. ${ }^{158,159}$ Accordingly, electrons are easily excited from the HOMO of the p-doped molecule to the LUMO of the n-doped molecule, resulting in the generation of hole-electron pairs at the interface. They will dissociate into free charges under the induction of an electrical field, followed by the injection of electrons and holes into two adjacent emission units. 


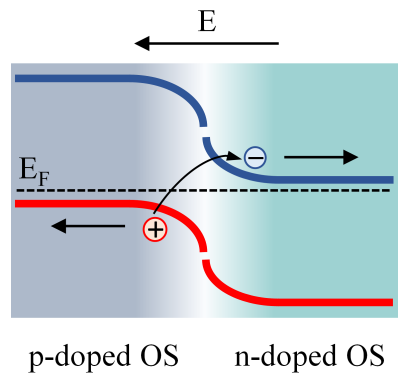

Figure 4.8 Schematic of the electron-hole generation and dissociation at the interface of a CGL.

\subsubsection{Doped layers in OPVs}

Different from the weak interaction in the emitting layer of OLEDs, it has been demonstrated that the donor and acceptor molecules can form CPX in the active layer of OPVs, which defines the CT state. It is an important concept that relating to the external quantum efficiency (EQE) and open-circuit voltage of the device. ${ }^{112,160}$ The relationship between $\mathrm{EQE}$ and the energy of the CT state $\left(\mathrm{E}_{\mathrm{CT}}\right)$ is determined by the following equations: ${ }^{160}$

$$
E Q E(E)=\frac{f}{E \sqrt{4 \pi \lambda k_{B} T}} \exp \left(\frac{-\left(E_{C T}+\lambda-E\right)^{2}}{4 \lambda k_{B} T}\right)
$$

where $f$ is proportional to the absorption strength of the CT state and the density of the donor/acceptor interfaces, $\lambda$ represents the reorganized energy of the CT state and E is the photon energy. Due to the low absorption of the CPX in OPVs, the $\mathrm{E}_{\mathrm{CT}}$ is usually derived by fitting the measured EQE spectrum using the above equation.

The application of doped OSs in other layers of OPVs is not as popular as in OLEDs. The charge transport and extraction from the active layer to the electrodes are relatively easy due to the narrower band gap of the molecules used in the active layer. Nevertheless, the doped OS is still used in certain scenarios. For most polymeric bulk heterojunction OPVs, the active layer is usually thick enough to ensure light absorption, which can be sandwiched between two electrodes with only a thin interlayer at each contact. In this case, doping is rarely observed in the device except for the electrode such as PEDOT:PSS and some cathode interlayers used in the inverted structure such as the $\mathrm{ZnO}$-polymer nanocomposite and BPhen: $\mathrm{Cs}_{2} \mathrm{CO}_{3} \cdot{ }^{161-163}$ Sometimes the interlayer molecules can also dope the active layer via charge transfer at the interface, which is an effective way to reduce the active layer/electrode contact resistance and improve the device performance. ${ }^{164-167}$ For 
instance, tetra-alkyl ammonium salts have been demonstrated to form effective doping for both fullerene and non-fullerene acceptors when used as a cathode interlayer. ${ }^{164,165}$
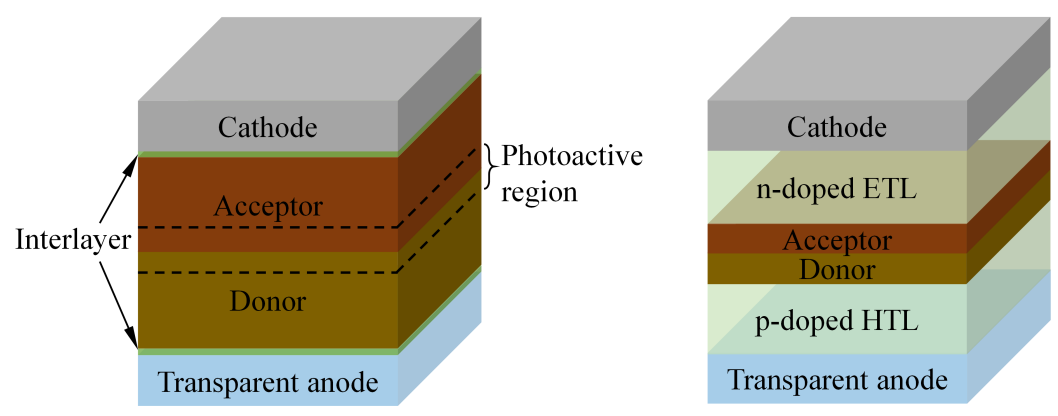

Figure 4.9 The traditional planar heterojunction OPV structure (left) and the p-i-n OPV structure with thin active layers and doped transport layers (right). The narrow photoactive region is located at both sides of the hetero interface, as marked by the dash line in the left figure.

For the small-molecular or planar heterojunction OPVs, only excitons formed near the hetero interface are able to dissociate into free charges and contribute to the photocurrent due to the short diffusion distance of the exciton in most OSs. Therefore, the active layer should not be too thick to ensure the absorption occurs at the photoactive region. Considering that the thin film may cause the short circuits and exciton recombination at the metallic contacts, transparent (large band gap) transport layers are commonly employed between the active layer and the electrodes. In order to improve the conductivity of the transport layer, doped OS is usually used to form a p-i-n structure, as shown in Figure 4.9. ${ }^{168-173}$ Similar to that in OLEDs, the doped transport layer is usually separated by a pure OS layer to avoid direct contact of the dopant with the active layer, and it can connect to the electrode without any interlayer. ${ }^{168,169}$ 


\section{Chapter 5 Photoelectron spectroscopy}

Photoelectron spectroscopy (PES) is a powerful experimental technique to measure the binding energies of electrons emitted from materials. It is based on the photoelectric effect discovered by Hertz in 1887 and theoretically explained by Einstein in 1905, which is a key step in the development of quantum mechanics. The first PES technique, X-ray photoelectron spectroscopy (XPS) was developed in 1957 to analyze the chemical environment of atoms via detecting the energy level shift of atomic core electrons. In 1961, ultraviolet photoelectron spectroscopy (UPS) was developed to study the valence energy levels and chemical bonding of e.g., firstly gas-phase molecules. After years of development, the application of PES technique has been significantly expanded, ${ }^{174}$ such as the analysis of the elemental composition, elemental ratio and chemical bonding of materials, the detection of element distribution and molecular orientation, the exploration of energy level alignment at the interface, etc. Compared to other analytical techniques, PES provides the useful combination of surface sensitivity, quantifiable elemental, and chemical information with a relatively non-destructive manner.

The characterization work of this thesis is mostly based on UPS and XPS. Thereinto, UPS experiments are performed in a home-designed spectrometer using monochromatized He I radiation with excitation energy of $21.22 \mathrm{eV}$. XPS is recorded by the Scienta-200 hemispherical analyzer using monochromatized $\mathrm{Al} \mathrm{K} \alpha$ radiation of $1486.6 \mathrm{eV}$ energy. All measurements were carried out with a base pressure lower than $1 \times 10^{-9}$ mbar to avoid significant electron collision and surface contamination. Another relevant surface technique, near edge X-ray adsorption fine structure spectroscopy (NEXAFS) is also used to characterize the molecular orientation, which is carried out at the FlexPES beamline of the $1.5 \mathrm{GeV}$ storage ring in MAX IV laboratory.

\subsection{Fundamentals of photoelectron spectroscopy}

The physical phenomenon behind PES is the photoelectric effect. As proposed by Einstein, the light shows discontinuous energy and is composed of energy quanta, i.e., photon, each with the energy $h v$, where $h$ is Planck's constant and $v$ is the light frequency. Photons with sufficient energy $(h v)$ can be absorbed by molecules and initiate ionization to eject electrons, known as photoelectrons $\left(\mathrm{e}^{-}\right)$. The process is expressed as:

$$
M_{0}+h v \rightarrow M^{+*}+e^{-}
$$


where $\mathrm{M}_{0}$ represents the neutral molecule in the ground state and $\mathrm{M}^{+*}$ is the molecular cation in the excited state. The kinetic energy $\left(\mathrm{E}_{\mathrm{k}}\right)$ of the photoelectron is determined by the following equation:

$$
E_{0}+h v=E^{+*}+E_{k}
$$

where $\mathrm{E}_{0}$ accounts for the total energy of the initial state (the molecule before photoionization) and $\mathrm{E}^{+*}$ is the total energy of the final state (ionized molecule after electron emission). The energy difference between $\mathrm{E}_{0}$ and $\mathrm{E}^{+*}$ is defined as electron binding energy, $\mathrm{E}_{\mathrm{B}}$. Therefore, the relationship between the kinetic energy $\mathrm{E}_{\mathrm{k}}$ and binding energy $\mathrm{E}_{\mathrm{B}}$ can be derived from equation 5.2 as:

$$
E_{B}=h v-E_{k}
$$

The electron binding energy of a certain photoelectron can be obtained by measuring the kinetic energy for a given photon energy. Recording the number of photoelectrons as a function of the binding energy yields the PES spectrum, as shown in Figure 5.1.

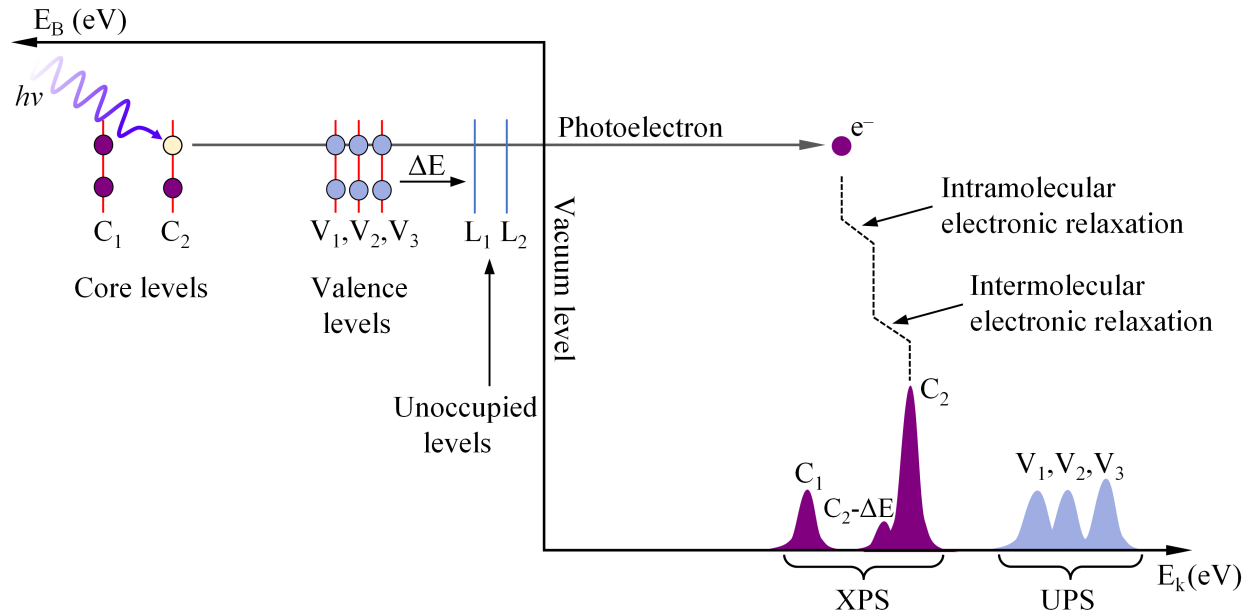

Figure 5.1 Schematic of photoemission process and the production of the PES spectrum including valence levels recorded by UPS and core levels recorded by XPS.

Electron binding energy is an important concept in PES measurements. It should be distinguished from any individual energy of electrons in an occupied valence level or core level because electrons are not independent but share simultaneously the total energy of the system. Removing an electron will bring some other changes to the molecule, such as the relaxation of electrons, which may affect the final binding energy. During the 
photoemission process, the photoelectron leaves the molecule generally within $10^{-15} \mathrm{~s}$. The relaxation time of the remaining electrons to screen the hole is about $10^{-16} \mathrm{~s}$, while the nuclei geometric relaxation needs roughly $10^{-13} \mathrm{~s} .{ }^{175}$ Hence, electrons are able to fully relaxed, whereas the nuclei are frozen during this process, which means that the PES measurement will overestimate the formation energy of a fully relaxed positive polaron and the binding energy thereof. In solid films, surrounding molecules also affect the binding energy via electronic polarization and further lower the formation energy of positive polaron. While lattice relaxation has a similar time-scale to nuclei relaxation and thus is not taken into account as well. As shown in Figure 5.1, electronic relaxation is reflected in the PES spectra with increased kinetic energy compared to electrons from the initial state of an isolated neutral molecule, however, the nuclei relaxation is not involved. The influence of polarization energy on IP and EA has already discussed in Figure 2.6.

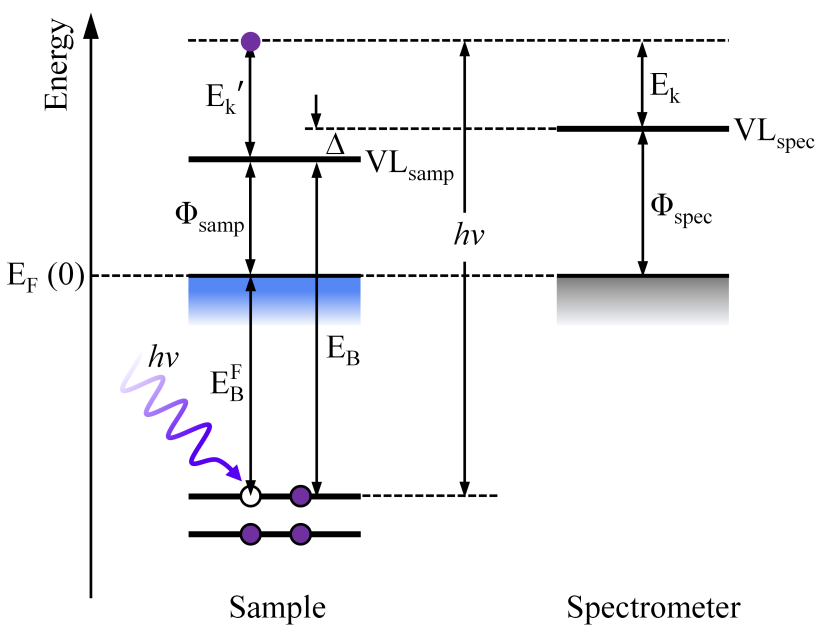

Figure 5.2 Schematic explanation of relevant energy terms in PES measurement of solid surfaces. Left panel is the sample and right panel is the spectrometer. VL is the vacuum level. $\mathrm{E}_{\mathrm{B}}^{\mathrm{F}}$ is the binding energy relative to the Fermi level.

In the PES measurement of molecule solids, Fermi level is used as the reference rather than vacuum level. The Fermi level of the sample is aligned to that of the spectrometer by charge transfer and both are set to $0 \mathrm{eV}$. In this context, the sample needs to be conductive and in good contact with the spectrometer. The vacuum level shifts $(\Delta)$ as the work function of the sample changes $\left(\Phi_{\text {samp }}\right)$, as shown in Figure 5.2. In this case, the equation 5.3 can be amended as:

$$
E_{B}^{F}+\Phi_{\text {samp }}+\Delta=h v-E_{k}
$$


shorted as

$$
E_{B}^{F}=h v-E_{k}-\Phi_{\text {spec }}
$$

where $\mathrm{E}_{\mathrm{B}}^{\mathrm{F}}$ is the binding energy relative to the Fermi level, $\Phi_{\text {spec }}$ is the work function of the spectrometer, $\mathrm{E}_{\mathrm{k}}$ ' and $\mathrm{E}_{\mathrm{k}}$ account for the kinetic energy when the photoelectron leaves the sample and reaches the analyzer after accelerating or retarding by the electron lens. When photon energy and work function of the spectrometer are known, the binding energy relative to the Fermi level can be obtained by measuring the kinetic energy of photoelectrons from the equation 5.4. Regardless of the work function of the sample, photoelectrons from a given core level under the same photon excitation will always appear in the spectrometer with the same kinetic energy.

When the sample is poorly conductive or in bad contact with the spectrometer, the holes produced during the photoemission process cannot be neutralized. In this case, the assumption of the Fermi level alignment between the sample and the spectrometer is invalid. Holes will accumulate in the sample as it is measured, which is usually referred to as charging. As a result, the Fermi level of the sample is shifted, and the binding energy of the measured spectrum is always higher than the case with sufficient electron compensation. Therefore, we need to ensure the sample has good conductivity and is in good contact with the spectrometer before the measurement. Otherwise, using an external electron source, such as an electron flow gun is helpful to improve the charge neutralization.

\subsection{Ultraviolet photoelectron spectroscopy (UPS)}

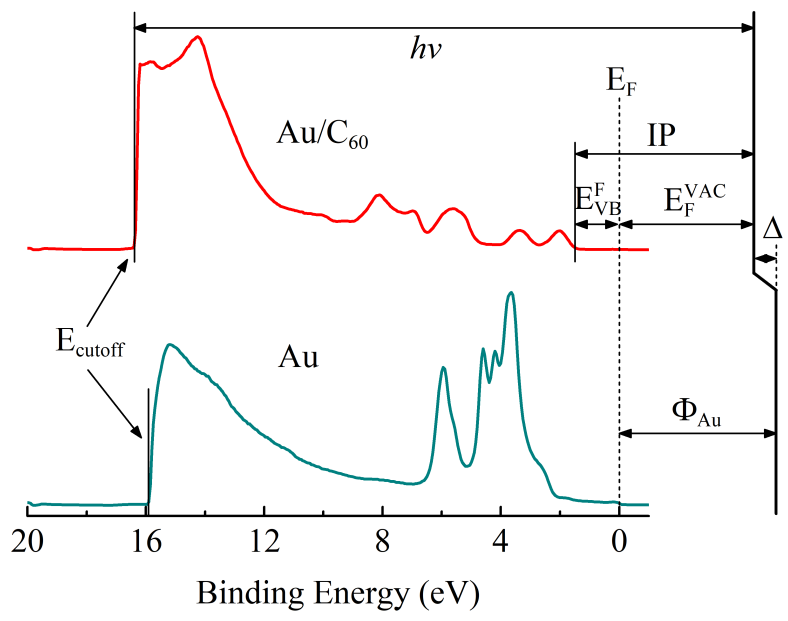

Figure 5.3 UPS spectrum of the sample $\mathrm{Au} / \mathrm{C}_{60}$ (right) as well as the $\mathrm{Au}$ substrate (left). The energy of the photon source $h v=21.22 \mathrm{eV}$. 
By using low-energy radiation, e.g., $21.22 \mathrm{eV}$ or $40.8 \mathrm{eV}$ for the typical helium discharge photon source, UPS usually probes the valence electronic states of materials with high resolution. We analyze the UPS spectrum by using a fullerene sample deposited on the metal substrate $(\mathrm{Au})$, as shown in Figure 5.3, where all information provided by the spectrum are clearly marked. As we discussed in the previous section, the UPS spectra are aligned to Fermi level, which is $0 \mathrm{eV}$ labeled in the figure. The valence feature begins to appear at $\mathrm{E}_{\mathrm{VB}}^{\mathrm{F}}$ below the Fermi level, which determines the hole injection barrier from the metal to the OS. The left features with high binding energy are contributed by deeper states and the most pronounced signal in the far left originates from the inelastically scattered electrons. From the secondary-electron cutoff $\left(\mathrm{E}_{\text {cutoff }}\right)$, we can calculate the work function $(\Phi)$ of the sample by the following equation:

$$
\Phi=E_{F}^{V A C}=h v-E_{\text {cutoff }}
$$

Work function is a property of the film surface that determines the minimum energy required to remove electrons from the material to the vacuum. It is an important concept in the surface and interface research. The work function modification by the OS, i.e., $\Delta$ determines the energy level alignment at the metal/OS interface. The ionization potential (IP) of the OS is derived from the sum of the work function and the HOMO onset, corresponding to the energy difference between the HOMO level and vacuum level.

$$
\mathrm{IP}=E_{F}^{V A C}+E_{V B}^{F}
$$

The IP is independent of the work function of the substrate. Its value obtained by this method is usually overestimated since the nuclei relaxation is not considered. From UPS we cannot get the unoccupied levels due to the absence of electrons. To study the unoccupied levels and get the EA, the inverse photoelectron spectroscopy (IPES) is required, which is not involved here. More information about IPES can be found in the review article and textbook. ${ }^{176,177}$

\subsection{X-ray photoelectron spectroscopy (XPS)}

Different from UPS, the commonly used photon sources in XPS are $\mathrm{K} \alpha \mathrm{X}$-ray from $\mathrm{Al}$ or $\mathrm{Mg}$ anodes, whose primary lines have energies of $1253.6 \mathrm{eV}$ and $1486.6 \mathrm{eV}$, respectively. The high photon energy enables the probe of the deep-lying core levels as well as the valence band. However, the transition probability of the valence electrons is very small radiated by such high photon energy. Even though the valence electrons are ionized by such photons, the photoelectrons will have ultrahigh kinetic energy, which is associated with the low resolution and the increase of the wave vector. 
Each element has a unique binding energy for a given core level, which enables the qualitative or even quantitative analysis of the chemical composition of the sample surface. For a given photon source, the peak intensity is determined by the atomic sensitivity factor (ASF) of the core level and the atom number. Under special conditions, such as the ambient pressure measurement, the change in pressure will also affect the final intensity. Some major concepts and their influence on the spectrum are introduced below.

\subsubsection{Spin-orbit splitting}

During the photoemission process, the removal of an electron from the core level leaves a single electron either spin-up or spin-down in the orbital and induces the spin-orbit coupling. As a result, the degeneracy is lifted and the core level is split into two sublevels when the angular quantum number is $l>0$, i.e., $p_{1 / 2}-p_{3 / 2}, d_{1 / 2}-d_{3 / 2}, f_{1 / 2}-f_{3 / 2}$. For $l=0$, i.e., the $s$ orbital, there is no spin-orbit splitting and only one peak in the spectrum. The theoretical area ratio of the two peaks is determined by the degeneracy $(2 j+1)$ of each sublevel, where $j$ accounts for the total angular momentum quantum number and is equal to the sum of the angular and the spin quantum number $\left(l+m_{s}\right)$, as shown in Table 5.1. The energy splitting between the two sublevels depends on the average radius of the orbital involved. In general, it increases with atomic number when the principle and angular quantum numbers ( $n$ and $l$ ) are constant and decreases with the increase of $l$ when $n$ is constant.

Table 5.1 Spin-orbit splitting of each orbital.

\begin{tabular}{|c|c|c|c|c|c|}
\hline Orbital & $l$ & $m_{s}$ & $\begin{array}{c}j \\
\left(l+m_{s}\right)\end{array}$ & $\begin{array}{c}\text { Degeneracy } \\
(2 j+1)\end{array}$ & $\begin{array}{c}\text { Area ratio } \\
(2 j+1)\end{array}$ \\
\hline \multirow{2}{*}{$s$} & 0 & $+1 / 2,-1 / 2$ & $1 / 2$ & - & - \\
\hline \multirow{2}{*}{$p$} & \multirow{2}{*}{$d$} & $+1 / 2$ & $1 / 2$ & 2 & $\left(p_{1 / 2}: p_{3 / 2}\right)$ \\
\cline { 3 - 5 } & \multirow{2}{*}{$d$} & $-1 / 2$ & $3 / 2$ & 4 & $1: 2$ \\
\hline \multirow{2}{*}{$f$} & \multirow{2}{*}{3} & $+1 / 2$ & $3 / 2$ & 4 & $\left(d_{3 / 2}: d_{5 / 2}\right)$ \\
\cline { 3 - 5 } & \multirow{2}{*}{3} & $-1 / 2$ & $5 / 2$ & 6 & $\left(f_{5 / 2}: f_{7 / 2}\right)$ \\
\cline { 3 - 5 } & & $-1 / 2$ & $7 / 2$ & 8 & $3: 4$ \\
\hline
\end{tabular}

\subsubsection{Chemical shifts}

As mentioned above, one of the major applications of PES is to characterize the bonding state of the analyzed elements, which can be reflected by the change in binding energy. This energy difference caused by the bonding state is called chemical shift. A typical example, 
ethyl trifluoroacetate is composed of four types of carbon, corresponding to four distinct $\mathrm{C}$ 1s peaks with the same intensity, as shown in Figure 5.4. ${ }^{178}$ Therefore, the core level electrons are strongly affected by the bonding state even though only valence electrons that participate in the formation of chemical bonds. The physics behind it is that the valence electron density changes with the bonding state, which will further affect the screening to the hole created by photoemission. For instance, $\mathrm{C}$ atom in $\mathrm{CF}_{3}$ has the lowest valence electron density due to the high electronegativity of the bonded $\mathrm{F}$ atoms, resulting in the poorer screening of the core hole and smaller relaxation energy. According to the discussion of Figure 5.1, the measured kinetic energy of electrons will be reduced, corresponding to the high binding energy. On the contrary, the $\mathrm{C}$ atom in $\mathrm{CH}_{3}$ has the highest valence electron density and thus shows the lowest binding energy, while the $\mathrm{C}$ atoms attached to $\mathrm{O}$ show intermediate chemical shifts.

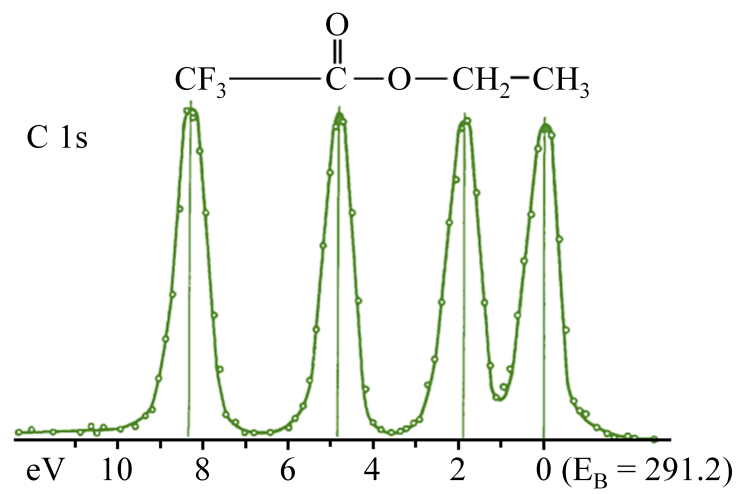

Figure 5.4 High-resolution C 1s core level spectrum of ethyl trifluoroacetate. Each peak corresponds to the carbon shown above. The spectrum is adapted from Ref. 178.

\subsubsection{Shake-up and shake-off satellites}

The most pronounced features in a PES spectrum are contributed by photoelectrons emitted to the vacuum level and left holes directly at the initial level, which are also mostly concerned in research. As shown in Figure 5.5a and b, the valence electrons with low binding energy can be ionized by low-energy photons and core level electrons require higher energy photons, corresponding to the UPS and XPS, respectively. However, in XPS measurement, the final states can also be affected by the valence levels through the interaction between the photoelectron and the valence electron. In Figure $5.5 \mathrm{c}$, the photoemission of a core level electron is accompanied by the excitation of a valence electron to the unoccupied level, producing shake-up satellites at the high energy side (see the peak 
$\mathrm{C}_{2}-\Delta \mathrm{E}$ in figure 5.1). When the valence electron is excited to the continuum states, then it forms the so-called shake-off satellites, as shown in Figure 5.5d.

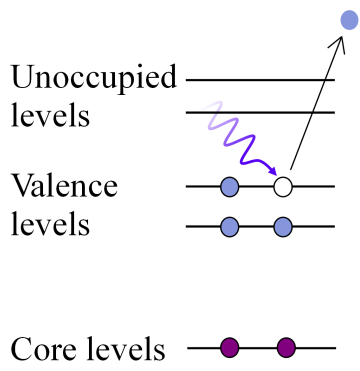

(a) on-set ionization

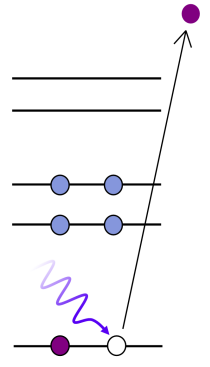

(b) core level ionization

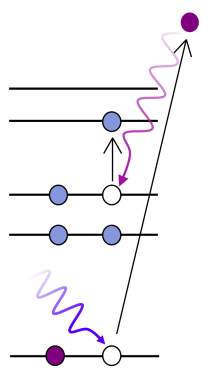

(c) shake-up

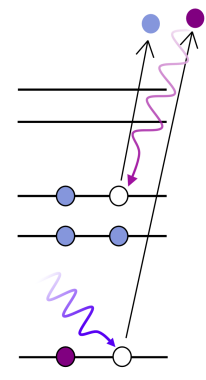

(d) shake-off

Figure 5.5 Schematic diagram of different final states. (a) and (b) are corresponding to the UPS and XPS, respectively. (c) and (d) refer to the formation of shake-up and shake-off satellites. 


\section{Chapter 6 Summary and outlook}

\subsection{Summary of papers}

\subsubsection{Paper I \\ Image-force effects on energy level alignment at electron transport material/cathode interfaces}

Yongzhen Chen, Xianjie Liu, Slawomir Braun, Ying Wang, and Mats Fahlman

Journal of Materials Chemistry C, 2020, 8, 173-179

In this paper, we analyzed the interface dipole of a typical electron transport material (ETM) bathophenanthroline (BPhen) and proposed a new insight into its formation mechanism. A series of conducting substrates with work function spanning from $3.8 \mathrm{eV}$ (Aluminum) to $5.7 \mathrm{eV}$ (UVO-Au) are employed to study the energy level alignment upon BPhen deposition. We observe the interfacial behavior follows the integer charge transfer (ICT) model with an additional displacement of $-1.4 \mathrm{eV}$, which means the vacuum level always upshifts an extra $1.4 \mathrm{eV}$ at the interface compared to the ideal ICT model, as shown in Figure 6.1.

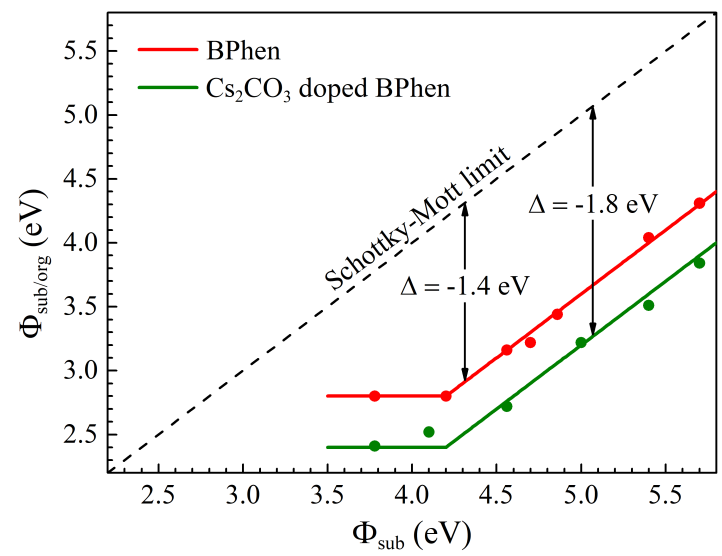

Figure 6.1 Work function of BPhen and BPhen:10 wt $\% \mathrm{Cs}_{2} \mathrm{CO}_{3}$ modified substrate $\left(\Phi_{\text {sub/org }}\right)$ changing with the work function of substrate $\left(\Phi_{\text {sub }}\right)$. 
This behavior is explained by the "double dipole step" modified ICT model. In detail, the interface dipole is composed of two parts: (i) a dipole caused by the integer charge transfer to get Fermi level alignment, only existing when $\Phi_{\text {sub }}<\mathrm{E}_{\mathrm{ICT}-}$ or $\Phi_{\text {sub }}>\mathrm{E}_{\mathrm{ICT}+}$, where $\mathrm{E}_{\mathrm{ICT}}$ is the pinning energy and has been discussed in chapter 3; (ii) an additional dipole potential created by the lone pairs of electrons and nuclei of the nitrogen atoms and their induced image charges when they are located close to the substrate surface. We find that the dipole will increase from $-1.4 \mathrm{eV}$ to $-1.8 \mathrm{eV}$ when BPhen is doped by $\mathrm{Cs}_{2} \mathrm{CO}_{3}$, as expected from the model as additional charged species are introduced to the interface. As a test of the UPS results and model, we fabricate the electron-only device and OLED using BPhen-modified ITO and BPhen: $\mathrm{Cs}_{2} \mathrm{CO}_{3}$-modified $\mathrm{ZnO}$ as cathodes, respectively. Both devices show excellent electron injection characteristics as predicted by the model and UPS results. This study provides a new interpretation for the interfacial behavior of ETMs/cathode. 


\subsubsection{Paper II}

\section{Understanding Interface Dipoles at an Electron Transport Material/Electrode Modifier for Organic Electronics}

Yongzhen Chen, Xianjie Liu, Slawomir Braun, and Mats Fahlman

ACS Applied Materials \& Interfaces, 2021, 13, 47218-47225

We have proposed that the energetics at the ETM/electrode interface can be explained by the "double dipole step" modified ICT model. Unlike electrolytes, who own free or covalently bonded ions that can act as the charged species to contribute to the double dipole potential, there are no obvious charged species in the small-molecule ETMs. We have suggested that the heteroatoms with lone pairs of electrons act as the charged species. In order to confirm this point, we investigate the influence of the number and orientation of the lone pair of electrons on the interface dipole through a series of pyridine derivatives, B2-B4PyMPM. They have similar chemical structures but different positions of nitrogen atoms in the pyridine rings, which are shown at the beginning of the chapter 2 . The interface dipoles measured by UPS show a uniform trend for the spin-coated films on various conducting substrates: $\Delta \Phi_{\mathrm{B} 2}>\Delta \Phi_{\mathrm{B} 3}>\Delta \Phi_{\mathrm{B} 4}$, whereas the vacuum-deposited films show similar interface dipoles for all three materials.

(a)

\section{(a)}

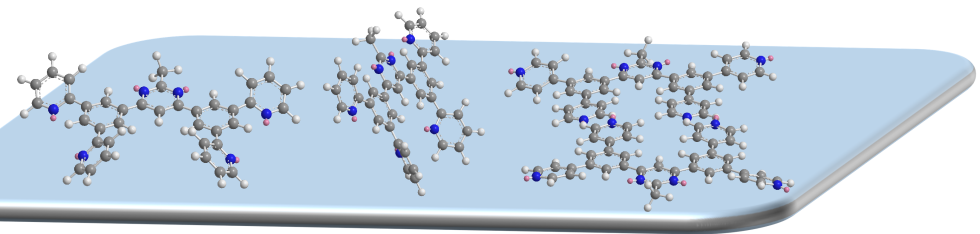

(b)

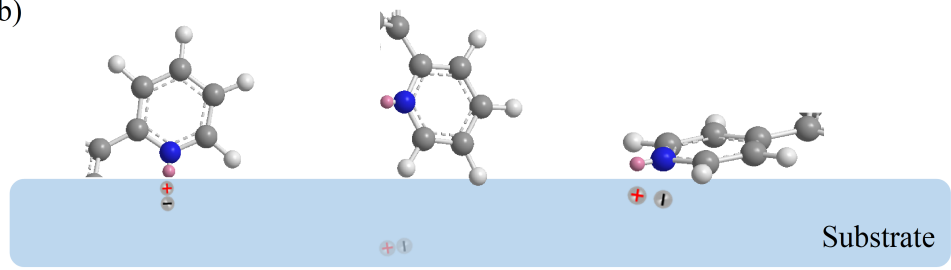

Figure 6.2 (a) Schematic of face-on (left) and edge-on (middle) oriented B2PyMPM and face-on oriented B4PyMPM dimer with hydrogen bonds (right). (b) Corresponding image charges formed on the surface of the substrate.

Through the $\mathrm{C}-\mathrm{H}$ stretching vibration and carbon core level analysis, we confirm the 
presence of weak hydrogen bonds $\mathrm{C}-\mathrm{H} \cdots \mathrm{N}$ both in the spin-coated and vacuum-deposited B3 and B4PyMPM films, which will occupy a part of lone pairs of electrons, as shown in Figure 6.2a. From the angle-resolved NEXAFS spectra, the preferential face-on orientation makes the interface dipole of B4PyMPM smaller than that of B3PyMPM in the spin-coated films. B2PyMPM has the largest areal density of lone electron pairs and optimal orientation, enabling it to achieve the largest interface dipole. However, in the vacuum-deposited films, the edge-on orientation of B2PyMPM prevents the lone electron pairs to move closer to the substrate and B3PyMPM has the same face-on orientation as B4PyMPM, resulting in the similar interface dipoles for all of them. These results indicate that the interface dipole formed by ETMs can be accurately explained by the "double dipole step" model, i.e., the nuclei and lone electron pairs of the heteroatoms contribute to the interface dipole, as shown in Figure 6.2b. 


\subsubsection{Paper III}

\section{Experimental and Theoretical Investigation into the Polaron Structure of K-Doped Polyfluorene Films}

Yongzhen Chen, Sarbani Ghosh, Xianjie Liu, Igor V. Zozoulenko, Mats Fahlman, and Slawomir Braun

The Journal of Physical Chemistry C, 2021, 125, 937-945

Understanding the formation mechanism and electronic structure of polarons is essential for the design of new OSs and electronic devices. Comprehensive studies on polarons in ndoped conjugated polymers are still rare, because the extreme air sensitivity of polarons requires an ultra-high vacuum environment during continuous doping and characterization. In this paper, by using a combined experimental approach based on the in-situ photoelectron spectroscopy and UV-vis-NIR absorption measurements, we explored the evolution of the electronic structure and optical transition with doping ratio in potassium-doped PFO films.

For both amorphous and crystalline films, a gap state is observed near the Fermi level in the UPS spectra upon K deposition, and new doubly-occupied states appear at the lower binding energy compared to the HOMO of the neutral PFO, accompanied by the significant reduction of the work function. In the UV-vis-NIR spectra, both films exhibit a prominent absorption band in the near-infrared (NIR, $<1.5 \mathrm{eV}$ ) region and a weak absorption band in the visible region $(\sim 2.2 \mathrm{eV})$. According to the time-dependent density functional theory (TD-DFT) calculation, these two new absorptions are assigned to the excitation from polaronic states to the conduction band and the excitation from the doubly-occupied valence band to the conduction band, respectively. The polaronic states simulated by the DFT show that polaron pairs rather than bipolarons are preferentially formed at high doping ratios. By comparing the amorphous and crystalline films, we find that the electronic structures of the polaron do not show much change with the conformation of the PFO chain, which is consistent with the calculation results. This is because the planar chain geometry in the crystalline film will be broken by the formation of polaron and transform to the amorphous conformation, as evidenced by the absence of the $\beta$-phase absorption in the crystalline film upon dedoping. 


\subsubsection{Paper IV \\ In-situ Spectroscopic and Electrical Investigations into Ladder-Type Conjugated Polymers Doped with Alkali Metals}

Yongzhen Chen, Han-Yan Wu, Chi-Yuan Yang, Xianjie Liu, Slawomir Braun, Simone Fabiano, and Mats Fahlman

Submitted

We have studied the polaronic properties of the classical conjugated polymer PFO and the influence of the conformation. In order to further investigate the dependence of the polaron and the geometry of the polymer chain, we turn our attention to the ladder-type conjugated polymers. They have a rigidly double-stranded and extended $\pi$-conjugated structure, which can maximum the delocalization of the polaron on the planar chain. However, their precise properties in chemical doping have remained largely unexplored. In this paper, we investigate the polaron formation in the ladder conjugated polymer polybenzimidazobenzophenanthroline (BBL) through the in-situ spectroscopic and electrical characterizations.

By using strong n-type dopant alkali metals, (e.g., K and Li), we successfully detect the polaronic states belowthe Fermi level and new absorption bands across the entire UV-vis-NIR regions. The evolutions of the UV-vis-NIR and UPS spectra upon sequential alkali metal doping provide a clear diagram for the frontier orbitals of multiply charged BBL. Through XPS analysis, the interaction between the BBL chain and $\mathrm{K}$ is figured out, where the electron distribution derived from the shift of each component after being charged agrees well with the reported DFT calculations. By comparison with the semi-ladder analogue $\mathrm{BBB}$, a larger (electron) polaron extension is observed on the BBL chain, resulting in the red shift of the NIR absorption band and less $\mathrm{K}$ required to reach a similar reduction level. From the different performances upon air exposure, we find that Li-BBL and K-BBB show more localized character than K-BBL, attributed to a more covalent bond for Li-BBL and ring torsion for K-BBB. Moreover, the electrical conductivity of BBL film doped by K shows a same trend as doped by small organic molecules (e.g., TDAE and benzyl viologen radical cation $\left(\mathrm{BV}^{\bullet+}\right)$ ) with a maximum value of $2.03 \mathrm{~S} \mathrm{~cm}^{-1},{ }^{128,179}$ while the case of $\mathrm{Li}$ doping shows two reverse stages during doping process and a higher conductivity, which has been observed only from the electrochemical reduction before. ${ }^{180}$ 


\subsection{Outlook}

The real interface behavior of the OSs/electrode is much more complicated than our modeling. We use the "double dipole step" model to explain the dipole potential formed by the small-molecule ETMs, which contain imine $\mathrm{N}$ in the pyridine rings. The same interfacial behavior has been observed in the tertiary aliphatic amines. Many other types of heteroatoms also contain lone pairs of electrons, such as the triarylamine, carbazole in the hole transport materials NPB and 4,4'-bis(N-carbazolyl)-1,1'-biphenyl (CBP), sulfur in the oligothiophene $\alpha-6 \mathrm{~T}$ and $\alpha$-quaterthiophene, and cyan, fluorine in the p-dopants HATCN and F4TCNQ. When they are deposited on a conducting substrate, the lone pairs in the heteroatoms always have the opportunity to contact the substrate surface and create the “double dipole step". However, most of these materials follow the ideal ICT model and no additional displacement is observed, which means the lack of a double dipole potential. Therefore, the relationship between the interface energetics and the chemical structure of OSs needs to be further investigated.

The electrical conductivity is dependent on free charges in the chemical doped OS. BBL doped with small-molecule dopants TDAE, BV ${ }^{\bullet+}$, and polymer PEI, bithiophenethiophene $\left(\mathrm{P}\left(\mathrm{g}_{4} 2 \mathrm{~T}-\mathrm{T}\right)\right)$ shows a comparable conductivity with respect to the alkali metal doping. However, the polaronic states in the organic doped BBL cannot be detected by UPS and the absorption signatures from highly reduced states are also absent in the UV-vis-NIR measurement. The spectroscopic results indicate that the doping efficiency of organic dopants is lower than that of alkali metals and the resulting free charges are much less, which contradicts the electrical characterization results. One related point is that bound ion pairs are formed after integer charge transfer without dissociating into free charges due to the strong Coulomb interaction in the organic-organic system. However, the contribution of bound ion pairs to electrical conductivity and optical absorption, and the correlation between bound ion pairs and $\mathrm{CT}$ states at the $\mathrm{D}-\mathrm{A}$ interface are still unclear and need to be further raveled out.

Furthermore, the energy level diagram of polarons is redrawn by the DFT calculation. As described in chapter 2, there are several changes in the new diagram, which could influence the understanding of polaron-related physical processes, such as the charge transport in the chemical doped films, integer charge transfer at the metal/organic or organic/organic interface. For instance, the replacement of the half-filled polaronic state by a spin-resolved state and the uncertainty of whether bipolarons or polaron pairs are formed at high doping levels will change the traditional interpretation of the conductivity evolution upon chemical doping. Therefore, it is necessary to investigate these processes in combination with the new diagram. 


\section{References}

1. S. Reineke et al, White organic light-emitting diodes with fluorescent tube efficiency. Nature, 459 (2009), 234-238.

2. M. C. Gather et al, White organic light-emitting diodes. Advanced Materials, 23 (2011), 233-248.

3. S.-J. Zou et al, Recent advances in organic light-emitting diodes: toward smart lighting and displays. Materials Chemistry Frontiers, 4 (2020), 788-820.

4. S. Wang et al, Towards high-power-efficiency solution-processed OLEDs: Material and device perspectives. Materials Science and Engineering: R: Reports, 140 (2020), 100547.

5. S. Günes et al, Conjugated polymer-based organic solar cells. Chemical Reviews, 107 (2007), 1324-1338.

6. A. W. Hains et al, Molecular semiconductors in organic photovoltaic cells. Chemical Reviews, 110 (2010), 6689-6735.

7. P. Cheng et al, Next-generation organic photovoltaics based on non-fullerene acceptors. Nature Photonics, 12 (2018), 131-142.

8. Y. Guo et al, Functional organic field-effect transistors. Advanced Materials, 22 (2010), 4427-4447.

9. L. Torsi et al, Organic field-effect transistor sensors: a tutorial review. Chemical Society Reviews, 42 (2013), 8612-8628.

10. C. Zhang et al, Organic field-effect transistor-based gas sensors. Chemical Society Reviews, 44 (2015), 2087-2107.

11. S. R. Forrest, The path to ubiquitous and low-cost organic electronic appliances on plastic. Nature, 428 (2004), 911-918.

12. T. Sekitani et al, Stretchable, large-area organic electronics. Advanced Materials, 22 (2010), 2228-2246.

13. E. Dauzon et al, Pushing the limits of flexibility and stretchability of solar cells: a review. Advanced Materials, 33 (2021), 2101469.

14. A. Salleo et al, Microstructural characterization and charge transport in thin films of conjugated polymers. Advanced Materials, 22 (2010), 3812-3838.

15. G. Heimel et al, Design of organic semiconductors from molecular electrostatics. Chemistry of Materials, 23 (2011), 359-377.

16. J. Hwang et al, Energetics of metal-organic interfaces: New experiments and assessment of the field. Materials Science and Engineering: R: Reports, 64 (2009), $1-31$.

17. M. Fahlman et al, Interfaces in organic electronics. Nature Reviews Materials, 4 (2019), 627-650. 
18. E. Zojer et al, The impact of dipolar layers on the electronic properties of organic/inorganic hybrid interfaces. Advanced Materials Interfaces, 6 (2019), 1900581.

19. I. E. Jacobs et al, Controlling molecular doping in organic semiconductors. Advanced Materials, 29 (2017), 1703063.

20. R. Schlaf et al, Photoemission spectroscopy of LiF coated Al and Pt electrodes. Journal of Applied Physics, 84 (1998), 6729-6736.

21. T. Mori et al, Electronic structure of 8-hydroxyquinoline aluminum/LiF/Al interface for organic electroluminescent device studied by ultraviolet photoelectron spectroscopy. Applied Physics Letters, 73 (1998), 2763-2765.

22. Y. Zhou et al, A universal method to produce low-work function electrodes for organic electronics. Science, 336 (2012), 327-332.

23. F. Huang et al, Novel electroluminescent conjugated polyelectrolytes based on polyfluorene. Chemistry of Materials, 16 (2004), 708-716.

24. H. Gommans et al, On the role of bathocuproine in organic photovoltaic cells. Advanced Functional Materials, 18 (2008), 3686-3691.

25. J.-H. Lee et al, A high performance inverted organic light emitting diode using an electron transporting material with low energy barrier for electron injection. Organic Electronics, 12 (2011), 1763-1767.

26. H. Fukagawa et al, Understanding coordination reaction for producing stable electrode with various low work functions. Nature Communications, 11 (2020), 3700.

27. C. B. Duke et al, Organic solids: is energy-band theory enough? Physics Today, $\mathbf{3 3}$ (1980), 42-48.

28. H. Ishii et al, Energy level alignment and interfacial electronic structures at organic/metal and organic/organic interfaces. Advanced Materials, 11 (1999), $605-625$.

29. N. Koch, Organic electronic devices and their functional interfaces. ChemPhysChem, 8 (2007), 1438-1455.

30. S. Braun et al, Energy-level alignment at organic/metal and organic/organic interfaces. Advanced Materials, 21 (2009), 1450-1472.

31. M. Fahlman et al, Electronic structure of hybrid interfaces for polymer-based electronics. Journal of Physics: Condensed Matter, 19 (2007), 183202.

32. J. L. Bredas et al, Polarons, bipolarons, and solitons in conducting polymers. Accounts of Chemical Research, 18 (1985), 309-315.

33. N. Lu et al, A review for polaron dependent charge transport in organic semiconductor. Organic Electronics, 61 (2018), 223-234.

34. G. Grancini et al, Hot exciton dissociation in polymer solar cells. Nature Materials, 12 (2013), 29-33.

35. V. Coropceanu et al, Charge transport in organic semiconductors. Chemical Reviews, 107 (2007), 926-952. 
36. S. J. Yoo et al, Charge transport in electrically doped amorphous organic semiconductors. Macromolecular Rapid Communications, 36 (2015), 984-1000.

37. M. Schwarze et al, Molecular parameters responsible for thermally activated transport in doped organic semiconductors. Nature Materials, 18 (2019), 242-248.

38. L. Zaikowski et al, Polarons, bipolarons, and side-by-side polarons in reduction of oligofluorenes. Journal of the American Chemical Society, 134 (2012), 10852-10863.

39. J. Yamamoto et al, Electronic and vibrational spectra of positive polarons and bipolarons in regioregular poly(3-hexylthiophene) doped with ferric chloride. The Journal of Physical Chemistry B, 119 (2015), 4788-4794.

40. D. T. Scholes et al, The effects of crystallinity on charge transport and the structure of sequentially processed $\mathrm{F}_{4} \mathrm{TCNQ}$-doped conjugated polymer films. Advanced Functional Materials, 27 (2017), 1702654.

41. I. Salzmann et al, Molecular electrical doping of organic semiconductors: fundamental mechanisms and emerging dopant design rules. Accounts of Chemical Research, 49 (2016), 370-378.

42. S. Hüfner, Photoelectron spectroscopy: principles and applications (Springer, 2003).

43. M. Pope et al, Electroluminescence in organic crystals. The Journal of Chemical Physics, 38 (1963), 2042-2043.

44. W. Helfrich et al, Recombination radiation in anthracene crystals. Physical Review Letters, 14 (1965), 229.

45. C. K. Chiang et al, Electrical conductivity in doped polyacetylene. Physical Review Letters, 39 (1977), 1098.

46. C. W. Tang et al, Organic electroluminescent diodes. Applied Physics Letters, 51 (1987), 913-915.

47. E. Menard et al, High-performance n- and p-type single-crystal organic transistors with free-space gate dielectrics. Advanced Materials, 16 (2004), 2097-2101.

48. S. J. Pookpanratana et al, Electronic properties and structure of single crystal perylene. Organic Electronics, 61 (2018), 157-163.

49. Y. Chen et al, N-doping-induced efficient electron-injection for high efficiency inverted organic light-emitting diodes based on thermally activated delayed fluorescence emitter. Journal of Materials Chemistry C, 5 (2017), 8400-8407.

50. K. H. Kim et al, Phosphorescent dye-based supramolecules for high-efficiency organic light-emitting diodes. Nature Communications, 5 (2014), 4769.

51. S.-F. Wu et al, White organic led with a luminous efficacy exceeding $100 \mathrm{~lm} \mathrm{~W}^{-1}$ without light out-coupling enhancement techniques. Advanced Functional Materials, 27 (2017), 1701314.

52. C.-H. Ji et al, Improving the performance of organic solar cells using an electron transport layer of B4PyMPM self-assembled nanostructures. Electronic Materials Letters, 11 (2015), 795-800. 
53. X. Shi et al, Relating chain conformation to the density of states and charge transport in conjugated polymers: the role of the $\beta$-phase in poly(9,9-dioctylfluorene). Physical Review X, 9 (2019), 021038.

54. C.-G. Zhan et al, Ionization potential, electron affinity, electronegativity, hardness, and electron excitation energy: molecular properties from density functional theory orbital energies. The Journal of Physical Chemistry A, 107 (2003), 4184-4195.

55. W. Warta et al, Hot holes in naphthalene: High, electric-field-dependent mobilities. Physical Review B, 32 (1985), 1172-1182.

56. W. Salaneck, Intermolecular relaxation energies in anthracene. Physical Review Letters, 40 (1978), 60-63.

57. M. Schwarze et al, Band structure engineering in organic semiconductors. Science, 352 (2016), 1446-1449.

58. H. Bässler, Localized states and electronic transport in single component organic solids with diagonal disorder. Physica Status Solidi (b), 107 (1981), 9-54.

59. W. Su et al, Soliton dynamics in polyacetylene. Proceedings of the National Academy of Sciences, 77 (1980), 5626-5629.

60. W. R. Salaneck et al, Electronic structure of conjugated polymers: consequences of electron-lattice coupling. Physics Reports, 319 (1999), 231-251.

61. S. Stafström et al, Evolution of the electronic structure of polyacetylene and polythiophene as a function of doping level and lattice conformation. Physical Review $B, 38$ (1988), 4180-4191.

62. G. Heimel, The optical signature of charges in conjugated polymers. ACS Central Science, 2 (2016), 309-315.

63. S. Ghosh et al, Electronic structures and optical absorption of n-type conducting polymers at different doping levels. The Journal of Physical Chemistry C, 123 (2019), $15467-15476$.

64. I. Zozoulenko et al, Polarons, bipolarons, and absorption spectroscopy of PEDOT. ACS Applied Polymer Materials, 1 (2018), 83-94.

65. Y. Chen et al, Experimental and theoretical investigation into the polaron structure of K-doped polyfluorene films. The Journal of Physical Chemistry C, 125 (2020), 937-945.

66. R. H. Friend et al, Electroluminescence in conjugated polymers. Nature, 397 (1999), 121-128.

67. W. H. Kim et al, Molecular organic light-emitting diodes using highly conducting polymers as anodes. Applied Physics Letters, 80 (2002), 3844-3846.

68. C. E. Small et al, Origin of enhanced hole injection in inverted organic devices with electron accepting interlayer. Advanced Functional Materials, 22 (2012), 3261-3266.

69. F. Wang et al, The role of molybdenum oxide as anode interfacial modification in the improvement of efficiency and stability in organic light-emitting diodes. Organic Electronics, 9 (2008), 985-993.

70. J. Blochwitz et al, Low voltage organic light emitting diodes featuring doped 
phthalocyanine as hole transport material. Applied Physics Letters, 73 (1998), 729-731.

71. J. Huang et al, Low-work-function surface formed by solution-processed and thermally deposited nanoscale layers of cesium carbonate. Advanced Functional Materials, 17 (2007), 1966-1973.

72. Y.-H. Kim et al, Polyethylene imine as an ideal interlayer for highly efficient inverted polymer light-emitting diodes. Advanced Functional Materials, 24 (2014), 3808-3814.

73. T.-L. Wu et al, Diboron compound-based organic light-emitting diodes with high efficiency and reduced efficiency roll-off. Nature Photonics, 12 (2018), 235-240.

74. A. Martínez-Otero et al, High-performance polymer solar cells using an optically enhanced architecture. Advanced Optical Materials, 1 (2013), 37-42.

75. J. Lee et al, Electron transport mechanism of bathocuproine exciton blocking layer in organic photovoltaics. Physical Chemistry Chemical Physics, 18 (2016), 5444-5452.

76. N. D. Lang et al, Theory of metal surfaces: work function. Physical Review B, 3 (1971), 1215-1223.

77. G. N. Derry et al, Recommended values of clean metal surface work functions. Journal of Vacuum Science \& Technology A: Vacuum, Surfaces, and Films, 33 (2015), 060801.

78. W. Osikowicz et al, Energetics at Au top and bottom contacts on conjugated polymers. Applied Physics Letters, 88 (2006), 193504.

79. Y. C. Chen et al, Dependence of rare-gas-adsorbate dipole moment on substrate work function. Physical Review B, 30 (1984), 7317-7319.

80. H. Krakauer et al, Initial oxidation of the $\mathrm{Al}(001)$ surface: Self-consistent electronic structure of clean $\mathrm{Al}(001)$ and $\mathrm{Al}(001)-\mathrm{p}(1 \times 1)$. Physical Review B, 23 (1981), 3859-3876.

81. Y. Maeno et al, Change in the work function of zirconium by oxidation at high temperatures and low oxygen pressures. Journal of the Chemical Society, Faraday Transactions, 87 (1991), 1399-1403.

82. K. J. Rietwyk et al, Universal work function of metal oxides exposed to air. Advanced Materials Interfaces, 6 (2019), 1802058.

83. J. Chen et al, Large on-off ratios and negative differential resistance in a molecular electronic device. Science, 286 (1999), 1550-1552.

84. X. Crispin et al, Characterization of the interface dipole at organic/metal interfaces. Journal of the American Chemical Society, 124 (2002), 8131-8141.

85. E. C. P. Smits et al, Bottom-up organic integrated circuits. Nature, 455 (2008), 956-959.

86. H. S. Kato et al, Strong hydrogen bonds at the interface between proton-donating and -accepting self-assembled monolayers on Au(111). Langmuir, 34 (2018), 2189-2197.

87. I. Campbell et al, Controlling schottky energy barriers in organic electronic devices using self-assembled monolayers. Physical Review B, 54 (1996), R14321. 
88. G. Heimel et al, The interface energetics of self-assembled monolayers on metals. Accounts of Chemical Research, 41 (2008), 721-729.

89. S. van Reenen et al, Origin of work function modification by ionic and amine-based interface layers. Advanced Materials Interfaces, 1 (2014), 1400189.

90. Q. Bao et al, Regular energetics at conjugated electrolyte/electrode modifier for organic electronics and their implications on design rules. Advanced Materials Interfaces, 2 (2015), 1500204.

91. Y. Chen et al, Image-force effects on energy level alignment at electron transport material/cathode interfaces. Journal of Materials Chemistry C, 8 (2020), 173-179.

92. S. Toyoshima et al, Electronic structure of bathocuproine on metal studied by ultraviolet photoemission spectroscopy. Japanese Journal of Applied Physics, 46 (2007), 2692-2695.

93. C. Tengstedt et al, Fermi-level pinning at conjugated polymer interfaces. Applied Physics Letters, 88 (2006), 053502.

94. S. Braun et al, Energy level alignment regimes at hybrid organic-organic and inorganic-organic interfaces. Organic Electronics, 8 (2007), 14-20.

95. L. Lindell et al, Self-assembled monolayer engineered interfaces: Energy level alignment tuning through chain length and end-group polarity. Journal of Electron Spectroscopy and Related Phenomena, 204 (2015), 140-144.

96. Q. Bao et al, The effect of oxygen uptake on charge injection barriers in conjugated polymer films. ACS Applied Materials \& Interfaces, 10 (2018), 6491-6497.

97. Q. Bao et al, Oxygen- and water-based degradation in [6,6]-phenyl-C $\mathrm{C}_{61}$-butyric acid methyl ester (PCBM) films. Advanced Energy Materials, 4 (2014), 1301272.

98. Q. Bao et al, Energetics at doped conjugated polymer/electrode interfaces. Advanced Materials Interfaces, 2 (2015), 1400403.

99. W. Salaneck et al, Photoelectron spectroscopy of iodine-doped polyacetylene. The Journal of Chemical Physics, 72 (1980), 3674-3678.

100. A. Pogantsch et al, Long lived photoexcitation dynamics in a dendronically substituted poly(fluorene). The Journal of Chemical Physics, 119 (2003), 6904-6910.

101. L. Groenendaal et al, Poly(3,4-ethylenedioxythiophene) and its derivatives: past, present, and future. Advanced Materials, 12 (2000), 481-494.

102. C. R. Bridges et al, Lewis acids and bases as molecular dopants for organic semiconductors. Journal of Physical Organic Chemistry, 33 (2020), e4077.

103. D. Kiefer et al, Double doping of conjugated polymers with monomer molecular dopants. Nature Materials, 18 (2019), 149-155.

104. N. Takeda et al, Nature and energies of electrons and holes in a conjugated polymer, polyfluorene. Journal of the American Chemical Society, 128 (2006), 16073-16082.

105. H. Mendez et al, Charge-transfer crystallites as molecular electrical dopants. Nature Communications, 6 (2015), 8560.

106. P. Pingel et al, Comprehensive picture of $p$-type doping of P3HT with the molecular acceptor F4TCNQ. Physical Review B, 87 (2013), 115209. 
107. T. J. Aubry et al, Tunable dopants with intrinsic counterion separation reveal the effects of electron affinity on dopant intercalation and free carrier production in sequentially doped conjugated polymer films. Advanced Functional Materials, 30 (2020), 2001800.

108. P. Pingel et al, Charge-transfer localization in molecularly doped thiophene-based donor polymers. The Journal of Physical Chemistry Letters, 1 (2010), 2037-2041.

109. B. X. Dong et al, Complex relationship between side-chain polarity, conductivity, and thermal stability in molecularly doped conjugated polymers. Chemistry of Materials, 33 (2021), 741-753.

110. D. A. Stanfield et al, Controlling the formation of charge transfer complexes in chemically doped semiconducting polymers. Chemistry of Materials, 33 (2021), 2343-2356.

111. J. B. Torrance, The difference between metallic and insulating salts of tetracyanoquinodimethone (TCNQ): how to design an organic metal. Accounts of Chemical Research, 12 (1979), 79-86.

112. K. Vandewal, Interfacial charge transfer states in condensed phase systems. Annual Review of Physical Chemistry, 67 (2016), 113-133.

113. J. J. Benson-Smith et al, Formation of a ground-state charge-transfer complex in polyfluorene//[6,6]-phenyl- $\mathrm{C}_{61}$ butyric acid methyl ester (PCBM) blend films and its role in the function of polymer/PCBM solar cells. Advanced Functional Materials, 17 (2007), 451-457.

114. L. Zhu et al, Prediction of remarkable ambipolar charge-transport characteristics in organic mixed-stack charge-transfer crystals. Journal of the American Chemical Society, 134 (2012), 2340-2347.

115. R. Sato et al, Asymmetrical hole/electron transport in donor-acceptor mixed-stack cocrystals. Journal of Materials Chemistry C, 7 (2019), 567-577.

116. J. Zhang et al, Sulfur-bridged annulene-TCNQ co-crystal: a self-assembled "molecular level heterojunction" with air stable ambipolar charge transport behavior. Advanced Materials, 24 (2012), 2603-2607.

117. J. Zhang et al, Recent progress on organic donor-acceptor complexes as active elements in organic field-effect transistors. Journal of Materials Chemistry C, 6 (2018), 3485-3498.

118. I. E. Jacobs et al, Quantitative dedoping of conductive polymers. Chemistry of Materials, 29 (2017), 832-841.

119. R. Haddon et al, Conducting films of $\mathrm{C}_{60}$ and $\mathrm{C}_{70}$ by alkali-metal doping. Nature, 350 (1991), 320-322.

120. M. Wang et al, High-mobility semiconducting two-dimensional conjugated covalent organic frameworks with $p$-type doping. Journal of the American Chemical Society, 142 (2020), 21622-21627.

121. M. Merkel et al, Photoemission study of the electronic structure of $\mathrm{C}_{60}$ and $\mathrm{K}_{x} \mathrm{C}_{60}$. Physical Review B, 47 (1993), 11470-11478. 
122. G. Greczynski et al, An experimental study of poly(9,9-dioctyl-fluorene) and its interfaces with Li, Al, and LiF. The Journal of Chemical Physics, 113 (2000), 2407-2412.

123. M. Fahlman et al, Experimental and theoretical studies of the electronic structure of Na-doped poly(para-phenylenevinylene). Chemical Physics Letters, 214 (1993), 327-332.

124. C. P. Cheng et al, Electronic structures of pristine and potassium-doped rubrene thin films. Organic Electronics, 14 (2013), 942-950.

125. R. Precht et al, Electronic structure and electrode properties of tetracyanoquinodimethane (TCNQ): a surface science investigation of lithium intercalation into TCNQ. Physical Chemistry Chemical Physics, 17 (2015), 6588-6596.

126. J. Meyer et al, Transition metal oxides for organic electronics: energetics, device physics and applications. Advanced Materials, 24 (2012), 5408-5427.

127. A. Nollau et al, Controlled $n$-type doping of a molecular organic semiconductor: Naphthalenetetracarboxylic dianhydride (NTCDA) doped with bis(ethylenedithio)tetrathiafulvalene (BEDF-TTF). Journal of Applied Physics, 87 (2000), 4340-4343.

128. S. Wang et al, Thermoelectric properties of solution-processed n-doped ladder-type conducting polymers. Advanced Materials, 28 (2016), 10764-10771.

129. S. Wang et al, A chemically doped naphthalenediimide-bithiazole polymer for n-type organic thermoelectrics. Advanced Materials, 30 (2018), e1801898.

130. L. Hu et al, An amidine-type n-dopant for solution-processed field-effect transistors and perovskite solar cells. Advanced Functional Materials, 27 (2017), 1703254.

131. C. K. Chan et al, Decamethylcobaltocene as an efficient n-dopant in organic electronic materials and devices. Organic Electronics, 9 (2008), 575-581.

132. B. Lüssem et al, Doped organic transistors operating in the inversion and depletion regime. Nature communications, 4 (2013), 2775.

133. S. Guo et al, N-doping of organic electronic materials using air-stable organometallics. Advanced Materials, 24 (2012), 699-703.

134. S. Olthof et al, Ultralow doping in organic semiconductors: evidence of trap filling. Physical Review Letters, 109 (2012), 176601.

135. X. Lin et al, Beating the thermodynamic limit with photo-activation of n-doping in organic semiconductors. Nature Materials, 16 (2017), 1209-1215.

136. B. D. Naab et al, Mechanistic study on the solution-phase n-doping of 1,3-dimethyl2-aryl-2,3-dihydro-1H-benzoimidazole derivatives. Journal of the American Chemical Society, 135 (2013), 15018-15025.

137. Y. Zeng et al, Doping mechanisms of N-DMBI-H for organic thermoelectrics: hydrogen removal vs. hydride transfer. Journal of Materials Chemistry A, 8 (2020), 8323-8328.

138. K. Shi et al, A novel solution-processable n-dopant based on 1,4-dihydropyridine motif for high electrical conductivity of organic semiconductors. Advanced Electronic 
Materials, 3 (2017), 1700164.

139. J. Gao et al, Aggregates promote efficient charge transfer doping of poly(3hexylthiophene). The Journal of Physical Chemistry Letters, 4 (2013), 2953-2957.

140. E. Lim et al, Thermoelectric properties of poly(3-hexylthiophene) (P3HT) doped with 2,3,5,6-tetrafluoro-7,7,8,8-tetracyanoquinodimethane ( $\left.\mathrm{F}_{4} \mathrm{TCNQ}\right)$ by vapor-phase infiltration. Chemistry of Materials, 30 (2018), 998-1010.

141. P. Y. Yee et al, Dopant-induced ordering of amorphous regions in regiorandom P3HT. The Journal of Physical Chemistry Letters, 10 (2019), 4929-4934.

142. R. Ghosh et al, Anisotropic polaron delocalization in conjugated homopolymers and donor-acceptor copolymers. Chemistry of Materials, 31 (2019), 7033-7045.

143. Y. Qi et al, A molybdenum dithiolene complex as $p$-dopant for hole-transport materials: a multitechnique experimental and theoretical investigation. Chemistry of Materials, 22 (2010), 524-531.

144. Y. Qi et al, Use of a high electron-affinity molybdenum dithiolene complex to p-dope hole-transport layers. Journal of the American Chemical Society, 131 (2009), 12530-12531.

145. J. Kim et al, Low work function $2.81 \mathrm{eV} \mathrm{Rb}_{2} \mathrm{CO}_{3}$-doped polyethylenimine ethoxylated for inverted organic light-emitting diodes. ACS Applied Materials \& Interfaces, 10 (2018), 18993-19001.

146. T. Earmme et al, Solution-processed, alkali metal-salt-doped, electron-transport layers for high-performance phosphorescent organic light-emitting diodes. Advanced Functional Materials, 22 (2012), 5126-5136.

147. Z. Wu et al, Management of singlet and triplet excitons: a universal approach to highefficiency all fluorescent WOLEDs with reduced efficiency roll-off using a conventional fluorescent emitter. Advanced Optical Materials, 4 (2016), 1067-1074.

148. H. Lee et al, Highly efficient green, blue, and white phosphorescent inverted organic light-emitting diodes by improving charge injection and balance. Journal of Materials Chemistry C, 5 (2017), 9911-9919.

149. W. Gao et al, Electronic structure and current injection in zinc phthalocyanine doped with tetrafluorotetracyanoquinodimethane: Interface versus bulk effects. Organic Electronics, 3 (2002), 53-63.

150. J. W. Kwon et al, Light-emitting characteristics of organic light-emitting diodes with the $\mathrm{MoO}_{x}$-doped NPB and $\mathrm{C}_{60} / \mathrm{LiF}$ layer. Thin Solid Films, 518 (2010), 6339-6342.

151. B. W. D'Andrade et al, Operational stability of electrophosphorescent devices containing $\mathrm{p}$ and $\mathrm{n}$ doped transport layers. Applied Physics Letters, 83 (2003), 3858-3860.

152. P. Wellmann et al, High-efficiency p-i-n organic light-emitting diodes with long lifetime. Journal of the Society for Information Display, 13 (2005), 393-397.

153. D.-S. Leem et al, Rubidium-carbonate-doped 4,7-diphenyl-1,10-phenanthroline electron transporting layer for high-efficiency p-i-n organic light emitting diodes. Electrochemical and Solid State Letters, 12 (2008), J8-J10. 
154. L. S. Liao et al, High-efficiency tandem organic light-emitting diodes. Applied Physics Letters, 84 (2004), 167-169.

155. T.-Y. Cho et al, Microcavity two-unit tandem organic light-emitting devices having a high efficiency. Applied Physics Letters, 88 (2006), 111106.

156. Q.-D. Ou et al, Photoemission spectroscopy study on interfacial energy level alignments in tandem organic light-emitting diodes. Journal of Electron Spectroscopy and Related Phenomena, 204 (2015), 186-195.

157. D. Zhao et al, Progress on material, structure and function for tandem organic lightemitting diodes. Organic Electronics, 51 (2017), 220-242.

158. M. Kröger et al, Temperature-independent field-induced charge separation at doped organic/organic interfaces: Experimental modeling of electrical properties. Physical Review B, 75 (2007), 235321.

159. M. Terai et al, Electric-field-assisted bipolar charge generation from internal charge separation zone composed of doped organic bilayer. Applied Physics Letters, 90 (2007), 083502.

160. K. Vandewal et al, Efficient charge generation by relaxed charge-transfer states at organic interfaces. Nature Materials, 13 (2014), 63-68.

161. Z.-Q. Xu et al, Efficient inverted polymer solar cells incorporating doped organic electron transporting layer. Organic Electronics, 13 (2012), 697-704.

162. C. E. Small et al, High-efficiency inverted dithienogermole-thienopyrrolodionebased polymer solar cells. Nature Photonics, 6 (2012), 115-120.

163. N. Wu et al, Zinc oxide: Conjugated polymer nanocomposite as cathode buffer layer for solution processed inverted organic solar cells. Solar Energy Materials and Solar Cells, 141 (2015), 248-259.

164. C.-Z. Li et al, Doping of fullerenes via anion-induced electron transfer and its implication for surfactant facilitated high performance polymer solar cells. Advanced Materials, 25 (2013), 4425-4430.

165. R. Wang et al, A universal approach for optimizing charge extraction in electron transporting layer-free organic solar cells via lewis base doping. Journal of Materials Chemistry A, 7 (2019), 25808-25817.

166. R. Wang et al, High efficiency non-fullerene organic solar cells without electron transporting layers enabled by Lewis base anion doping. Nano Energy, 51 (2018), 736-744.

167. Q. Kang et al, A printable organic cathode interlayer enables over $13 \%$ efficiency for 1-cm² organic solar cells. Joule, 3 (2019), 227-239.

168. B. Maennig et al, Organic $p$-i-n solar cells. Applied Physics A, 79 (2004), 1-14.

169. J. Drechsel et al, High efficiency organic solar cells based on single or multiple PIN structures. Thin Solid Films, 451-452 (2004), 515-517.

170. J. Drechsel et al, MIP-type organic solar cells incorporating phthalocyanine/fullerene mixed layers and doped wide-gap transport layers. Organic Electronics, 5 (2004), $175-186$. 
171. J. Drechsel et al, Efficient organic solar cells based on a double $p-i-n$ architecture using doped wide-gap transport layers. Applied Physics Letters, 86 (2005), 244102.

172. K. Schulze et al, Efficient vacuum-deposited organic solar cells based on a new lowbandgap oligothiophene and fullerene $\mathrm{C}_{60}$. Advanced Materials, 18 (2006), 2872-2875.

173. A. Mishra et al, Small molecule organic semiconductors on the move: promises for future solar energy technology. Angewandte Chemie International Edition, 51 (2012), 2020-2067.

174. G. Greczynski et al, X-ray photoelectron spectroscopy: Towards reliable binding energy referencing. Progress in Materials Science, 107 (2020), 100591.

175. M. Fahlman et al, Photoelectron spectroscopy and modeling of interface properties related to organic photovoltaic cells. Journal of Electron Spectroscopy and Related Phenomena, 190 (2013), 33-41.

176. H. Yoshida, Principle and application of low energy inverse photoemission spectroscopy: A new method for measuring unoccupied states of organic semiconductors. Journal of Electron Spectroscopy and Related Phenomena, 204 (2015), 116-124.

177. T. Fauster, V. Dose, Inverse photoemission spectroscopy. Chemistry and physics of solid surfaces VI (Springer, 1986), pp. 483-507.

178. U. Gelius et al, A high resolution esca instrument with X-ray monochromator for gases and solids. Journal of Electron Spectroscopy and Related Phenomena, 2 (1973), 405-434.

179. T. L. D. Tam et al, High-performance \& thermally stable n-type polymer thermoelectrics based on a benzyl viologen radical cation-doped ladder-type conjugated polymer. Journal of Materials Chemistry A, 9 (2021), 11787-11793.

180. T. Yohannes et al, Multiple electrochemical doping-induced insulator-to-conductor transitions observed in the conjugated ladder polymer polybenzimidazobenzophenanthroline (BBL). The Journal of Physical Chemistry B, 104 (2000), 9430-9437. 
Publications 


\section{Papers}

The papers associated with this thesis have been removed for copyright reasons. For more details about these see:

https://doi.org/10.3384/9789179290627 


\section{FACULTY OF SCIENCE AND ENGINEERING}

Linköping Studies in Science and Technology, Dissertation No. 2181, 2021 Department of Science and Technology

Linköping University

SE-581 83 Linköping, Sweden

www.liu.se

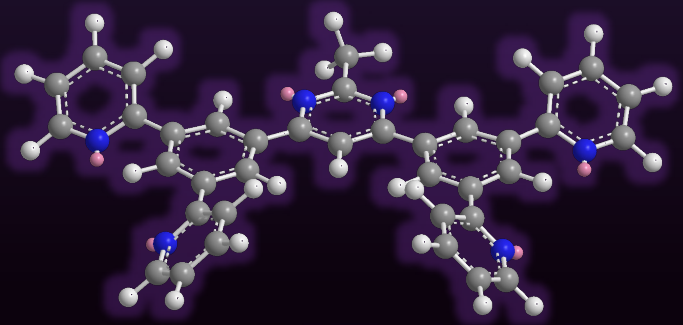

